Subscriber access provided by King Abdullah University of Science and Technology Library

\title{
Article
}

\section{Tuning the Electronic Properties of Hexagonal Two-Dimensional GaN Monolayers via Doping for Enhanced Optoelectronic Applications}

\author{
Naresh Alaal, and Iman S Roqan
}

ACS Appl. Nano Mater., Just Accepted Manuscript • DOI: 10.1021/acsanm.8b01852 • Publication Date (Web): 21 Dec 2018

Downloaded from http://pubs.acs.org on December 26, 2018

\section{Just Accepted}

"Just Accepted" manuscripts have been peer-reviewed and accepted for publication. They are posted online prior to technical editing, formatting for publication and author proofing. The American Chemical Society provides "Just Accepted" as a service to the research community to expedite the dissemination of scientific material as soon as possible after acceptance. "Just Accepted" manuscripts appear in full in PDF format accompanied by an HTML abstract. "Just Accepted" manuscripts have been fully peer reviewed, but should not be considered the official version of record. They are citable by the Digital Object Identifier (DOI®). "Just Accepted" is an optional service offered to authors. Therefore, the "Just Accepted" Web site may not include all articles that will be published in the journal. After a manuscript is technically edited and formatted, it will be removed from the "Just Accepted" Web site and published as an ASAP article. Note that technical editing may introduce minor changes to the manuscript text and/or graphics which could affect content, and all legal disclaimers and ethical guidelines that apply to the journal pertain. ACS cannot be held responsible for errors or consequences arising from the use of information contained in these "Just Accepted" manuscripts. 


\title{
Tuning the Electronic Properties of Hexagonal Two-Dimensional GaN Monolayers via Doping for Enhanced Optoelectronic Applications
}

\author{
Naresh Alaal and Iman S. Roqan* \\ *Email: iman.roqan@kaust.edu.sa
}

Physical Sciences and Engineering Division, King Abdullah University of Science and Technology, Thuwal 23955-6900, Saudi Arabia

We explore structural, electronic and magnetic properties of two-dimensional (2D) gallium nitride (GaN) monolayer (ML) doped with different elements belonging to the groups III-VI, using density-functionaltheory (DFT) with the Perdew-Burke-Ernzerhof (PBE) functional and the screened hybrid functional (HSE06) approaches as well as molecular dynamics (MD) simulations. Dopant interactions in Ga- and Nrich environments are investigated by varying their concentrations from $1.38 \%$ to $5.5 \%$. Our calculations reveal that oxygen and aluminium impurities are the most preferred candidates under Ga- and N-rich conditions, respectively. The electronic structure studies indicate that dopants containing an even number of valence electrons introduce magnetic behavior with spin-polarized properties, or n-type conductivity with nonmagnetic features, depending on the stoichiometric III/V ratio during growth. Dopants with an odd number of valence electrons modify the GaN ML band structure from indirect to direct bandgap at the $\Gamma$ point, depending on dopant types at different III/V ratios as well as substitutional site. The calculated charge transfer explains the dopants' influence on the band structure and bond nature. The HSE calculations of doped g-GaN MLs show a $0.23-1.48 \mathrm{eV}$ increase in the band gaps including the spin polarized band structures when compared with their PBE values. MD calculations suggest high structural stability at high growth temperatures. Such dopant-induced modifications in structural and physical properties of 2D GaN ML could potentially allow use of this material in diverse electronic, optoelectronic and spintronic applications.

\section{INTRODUCTION}

It is well known that bulk III-nitride compoundssuch as GaN, AlN, InN and their alloys-are direct band gap semiconductors that are used for a vast range of practical and reliable optoelectronic and electronic applications, such as lasers, lightemitting diodes, and transistors ${ }^{1-3}$, including devices operating in harsh environments ${ }^{4}$, due to their unique mechanical and structural properties ${ }^{5-}$ 7. Moreover, the heterostructure of $\mathrm{GaN}$ nanosheets can potentially be beneficial in solar harvesting applications ${ }^{8}$. Similar to bulk IIInitrides, the two-dimensional (2D) GaN alloys have received tremendous research attention due to the possibility of tuning their optical properties ${ }^{9}$, as their band gap can be tuned by generating $\mathrm{GaN}, \mathrm{AlN}$ and $\mathrm{InN}$ alloys ${ }^{10}$. In the past, graphene was the first $2 \mathrm{D}$ material to be synthesized successfully ${ }^{11}$, and its remarkable properties have been subsequently confirmed ${ }^{12-15}$.
However, its use in manufacturing semiconducting devices is limited, as its zero band gap restricts photocurrent generation, hindering its use in related applications ${ }^{16}$. This has further motivated extensive research aimed at identifying a nanomaterial with a finite band gap that exhibits electronic properties similar to those of graphene ${ }^{15,17-22}$. As a result of these efforts, 2D $\mathrm{GaN}$ was recently proposed as a viable alternative to graphene for use in semiconductor devices. The main significance of 2D materials stems from their low dimensionality, and potentially beneficial electronic properties for novel devices when compared with their three-dimensional (3D) counterparts ${ }^{23}$.

2D GaN was first synthesized by Balushi et al. by migration-enhanced encapsulated growth technique ${ }^{24}$. However, experimental reports on 2D $\mathrm{GaN}$ are scarce, as the crystal quality of such 2D material has not been optimized for practical applications compared to bulk. Theoretical 
predictions suggest that $\mathrm{GaN}$ material can be formed as a graphene-like hexagonal (g-GaN) monolayer (ML) ${ }^{25-27}$ with a strong covalent bond between $\mathrm{Ga}$ and $\mathrm{N}$ with $s p^{2}$ hybridization in the plane $^{26}$. The g-GaN structure is stable, with a planar geometry at room temperature, while its indirect band gap ${ }^{7}$ could be tuned from 1.8 to 3.5 $\mathrm{eV}$ by applying an external electric field ${ }^{28}$. Therefore, g-GaN is expected to exhibit optical absorption in the UV-Vis range ${ }^{29}$. Thus far, g-GaN ML has been studied theoretically by investigating the effect of chemical functionalization ${ }^{30}$, adsorption of gas molecules on its surface ${ }^{31}$, doping with adatoms and metals ${ }^{32-34}$, applying strain within the plane ${ }^{35}$, and introducing vacancies $^{36,37}$. However, the influence of using group III-VI elements as dopants, and the effect of their concentrations on g-GaN optical, electronic and magnetic properties have never been investigated in detail. Moreover, extant studies were mostly based on the Perdew-BurkeErnzerhof (PBE) and local density approximation (LDA) approaches. It is well known that PBE and LDA functionals underestimate the band gaps in semiconductors and are thus unreliable predictors of the interactions between the dopants and the host atoms. Hence, it is necessary to use a computationally expensive functional, such as Heyd-Scuseria-Ernzerhof (HSE06) hybrid functional, that can accurately predict band gap values in semiconductors ${ }^{38-40}$. The aforementioned shortcomings in the extant research have motivated this work, the aim of which is to explore different phenomena observed in doped g-GaN ML that would lead to a better understanding of the physics of its band structure and its properties.

In this work, we explore the effect of the dopants belonging to the groups III-VI on the structural, electronic, and magnetic properties of 2D g-GaN ML at different III/V ratios by using density functional theory (DFT), adopting both PBE and HSE approach as well as ab initio molecular dynamics (MD) simulations. To fully elucidate their influence, dopant concentrations varied from $1.38 \%$ to $5.5 \%$. We predict that the dopants with small radii will have the highest stability. Our findings reveal that the doped system exhibits diverse characteristics, depending on the dopant type, type of doping, dopant concentration, and the growth III/V ratio. We also show that the band gaps of doped systems corrected by hybrid functional give raise to a correction in the $0.23-1.48 \mathrm{eV}$ range, compared to that obtained from the PBE calculations. Such diverse electronic and magnetic properties of doped 2D GaN ML systems can be used in nanoelectronic and spintronic device applications.

\section{COMPUTATIONAL DETAILS}

All calculations conducted in this work were carried out using the DFT, as implemented in the Vienna $\mathrm{Ab}$ initio Simulation Package (VASP) ${ }^{41,42}$. For the exchange-correlation potential, we adopted the $\mathrm{PBE}^{43}$ functional with generalized gradient approximation (GGA), while the electron-ion interaction was described by projected augmented wave potentials. We have chosen $500 \mathrm{eV}$ as a kinetic energy cut-off for the plane wave basis sets. All the atoms in the unit cells were relaxed until the force on each atom declined below $0.01 \mathrm{eV} / \AA$, and the threshold energy convergence was set to $10^{-5} \mathrm{eV}$. A gamma centered $\mathrm{k}$-point mesh of $6 \times 6 \times 1$ was adopted to sample the first Brillouin zone. The hexagonal unit cell or supercell was considered in the $x y$ plane, whereas a vacuum of more than $15 \AA$ was adopted in the $z$ direction in order to preclude any interactions with adjacent cells. Bader ${ }^{44}$ method was employed to calculate the charge transfer between the dopant and the monolayer. In addition, we also utilized the HSE0 $6^{45}$ functional for the selected doped structures in order to provide supporting evidence for the band gap trends indicated by the PBE functional. The HSE06 functional combines $75 \%$ of the PBE exchange with $25 \%$ of the Hartree-Fock exchange. We carried out $a b$ initio MD simulations for selected doped configurations $(5 \times 5$ supercell $)$ using VASP, whereby the optimized geometries served as initial configurations. We adopted NoséHover thermostat ${ }^{46}$ method to control the temperatures chosen for the numerical investigation. The simulation time was set to $4 \mathrm{ps}$ with a step size of 0.5 fs. 
The dopants utilized in this work are (a) B, $\mathrm{Al}$, and In from group III; (b) $\mathrm{C}, \mathrm{Si}$, and $\mathrm{Ge}$ from group IV; (c) P, As and $\mathrm{Sb}$ from group V; and (d) $\mathrm{O}, \mathrm{S}$ and Se from group VI. We considered the dopants occupying either $\mathrm{Ga}$ or $\mathrm{N}$ sites and varied their concentrations from 1.38 to $5.5 \%$. In order to represent these dopant concentrations during modeling, one $\mathrm{Ga}$ or $\mathrm{N}$ atom was substituted with a dopant atom in the $3 \times 3,4 \times 4,5 \times 5$ and $6 \times 6 \mathrm{GaN}$ ML supercells. We divided this study into two parts, according to the environment, thus focusing first on (i) Ga-rich conditions, whereby one $\mathrm{N}$ atom in the supercell is substituted by a dopant; followed by (ii) N-rich conditions, where one Ga atom of the supercell is replaced by a dopant.

\section{RESULTS AND DISCUSSION}

\section{D GaN Monolayer}

First, we present the electronic properties of pristine 2D g-GaN ML to benchmark the methodology employed for the calculations. Furthermore, such calculation allows us to compare the results pertaining to doped ML with those obtained for pure g-GaN. The bond length of the Ga-N pair and the optimized lattice constant of planar g-GaN are $1.88 \AA$ and $3.25 \AA$, respectively. Fig. 1(a) depicts the calculated GaN ML band structures using PBE (black solid lines) and HSE (red solid lines) functionals. Unlike bulk GaN, both band structures show that the material possesses a semiconductor indirect band structure, as the valence band maximum (VBM) occurs between the highly symmetric Brillouin zone points, $\mathrm{K}$ and $\Gamma$, whereas the conduction band minimum $(\mathrm{CBM})$ coincides with the $\Gamma$ point. The PBE calculations result in a pure g-GaN ML band gap of $1.94 \mathrm{eV}$, whereas, when the band gap was corrected by HSE calculation, an energy gap of $3.21 \mathrm{eV}$ was obtained. Both band gap values are consistent with the previously reported results ${ }^{30-}$ 36,47-49. Moreover, the pure g-GaN ML exhibits nonmagnetic behavior. We also present the projected density of states (PDOS) of the pure 2D g-GaN ML in Fig. 1(b) in order to elucidate the individual atomic contributions. In addition, Fig. 1(b) shows that the VBM originates from the $p$ orbitals of $\mathrm{N}$ atoms, while $s$ orbitals of Ga atoms contribute to the CBM.
Electronic and magnetic properties of doped gGaN ML under Ga-rich conditions

First, we investigate the geometric structures of doped 2D g-GaN MLs. Here, we study the effect of different dopants (at $3.125 \%$ concentration) on the structural and electronic properties of g-GaN $\mathrm{ML}$, and find that similar trends are observed at other concentrations. Fig. 1(c) shows the atomic structure of g-GaN substitutionally doped with $\mathrm{X}$ at the $\mathrm{N}$ site under Ga-rich conditions, where $\mathrm{X}=$ $\mathrm{B}, \mathrm{C}, \mathrm{Al}, \mathrm{In}, \mathrm{Si}, \mathrm{Ge}, \mathrm{P}, \mathrm{As}, \mathrm{Sb}, \mathrm{O}, \mathrm{S}$, and Se. In this case, the dopant has three Ga atoms as the nearest neighbors. We show that none of the dopants induces any significant structural deformations in the g-GaN planar crystal structure. However, the bond distances surrounding the dopants are modified due to their atomic size and electronegativity, as well as the densities and affinities of electrons in each atom compared to $\mathrm{N}$, which can affect the band structure of the material. Similar behavior was reported for other 2D MLs ${ }^{40}$ doped with group III-V impurities. The calculated bond lengths of $\mathrm{Ga}-\mathrm{X}$ in the doped g-GaN MLs are shown in Table 1.

Among all the bond lengths, at $1.94 \AA$, $\mathrm{Ga}-\mathrm{C}$ and $\mathrm{Ga}-\mathrm{O}$ bonds are the shortest, as $\mathrm{C}$ and $\mathrm{O}$ have small atomic size. However, this value is slightly higher than the bond length of pure g-GaN ML because their atomic size and electronegativity are slightly different when compared to $\mathrm{N}$. The $\mathrm{Ga}-\mathrm{In}$ and $\mathrm{Ga}-\mathrm{Sb}$ pairs have the greatest bond length $(2.38$ and $2.37 \AA$ ) due to the large atomic size of these atoms compared to other dopants, introducing tensile strain. The $\mathrm{Ga}-\mathrm{B}$ bond length in the doped g-GaN $(2.02 \AA)$ is slightly shorter than that of the doped bulk GaN $(2.08 \AA)^{50}$. This difference is attributed to the elongated bond along the $c$-axis compared to that in the $a-b$ plane in bulk GaN, which is absent from 2D GaN. In general, the data presented in Table 1 shows that the bond length increases as the atomic size of the dopant increases. Similar trends were observed in experimental results pertaining to bulk $\mathrm{GaN}$ doped with different elements ${ }^{50}$. These observations are useful for tuning the band gap of the materials. 
To understand the effects of doping on the electronic structure and related properties of doped g-GaN in Ga-rich environment, we calculated the band structures and PDOS, which are depicted in Fig. 2 and Fig. 3, respectively. The band gap values of doped g-GaN (by replacing $\mathrm{N}$ atoms with $\mathrm{X}$ dopants), including the corrected HSE values, are shown in Table 1. For the group III dopants, the band structures of GaN doped with $\mathrm{B}, \mathrm{Al}$ and In are depicted in Fig. 2(a)-2(c), respectively. Unlike the pure g-GaN ML, the B-, Al-, and In-doped systems have VBM located at the $\Gamma$ point. Additional energy levels emerge within the band gaps when g-GaN is doped with group III elements, resulting in a reduction in the band gap energy. Such modification in the band gap energy is expected, as the nearest neighbors surrounding the dopants introduce an $\mathrm{X}-\mathrm{Ga}$ metal phase. Although these dopants belong to the same group, they induce different modifications in the nature of the band gap structure. When B and In are used as dopants, they transform the band gap structure of $\mathrm{g}-\mathrm{GaN}$ from indirect to direct band gap, as its VBM and CBM occur at the same symmetric point, $\Gamma$, as shown in Fig. 2(a). However, Al dopant shifts the CBM slightly away from the $\Gamma$ point, which results in an indirect band gap. The charge transfer is calculated by using the formula ${ }^{40} \delta q=\rho_{d}-Z_{d}$, where $Z_{d}$ is the charge located on the dopant atom in vacuum and $\rho_{\mathrm{d}}$ is the Bader charge of the dopant. In this expression, a positive/negative $\delta q$ sign denotes electron gain/loss. The charge transfer values (denoted as $\delta q$ in Table 1) indicate that the electron affinity in $\mathrm{B}$ atoms (positive $\delta \mathrm{q}$ ) is greater than that for $\mathrm{Al}$ and In atoms (as indicated by negative $\delta q$ ), as B atoms exhibit greater electronegativity than $\mathrm{Ga}^{51}$, which can influence the nature of the band structure.

The corresponding PDOS of the B-, Al-, and In-doped MLs are calculated in order to determine the contribution of individual dopants to the VBM and CBM. The PDOS illustrated in Fig. 3(a)-3(c) shows that the CBMs of g-GaN doped with all group III elements have a significant contribution from $p$ orbitals of these dopants. However, the $p$-orbital of $\mathrm{N}$ atoms provides a dominant contribution to VBMs, with some contributions from $\mathrm{B}$ and $\mathrm{Al} p$ orbitals.

The magnetic moments (MM) and the energy difference between the magnetic and nonmagnetic configurations, $\Delta E$, are shown in Table 1, indicating that group III-doped g-GaN MLs are nonmagnetic semiconductors under Garich conditions. With the exception of group IV dopants, elements from other groups investigated in this study show the same magnetic behavior. Indeed, when doped with group IV elements $(\mathrm{C}$, $\mathrm{Si}$, and $\mathrm{Ge}$ ), this doped g-GaN ML displays spinpolarized behavior, as the bands split into spin-up and spin-down channels exhibiting indirect semiconducting nature, as shown in Fig. 2(d)-2(f), respectively, with the two related band gaps presented in Table 1. In addition, when g$\mathrm{GaN}$ is doped with group IV elements other than $\mathrm{C}$, spin-up band gaps are larger than the spin-down band gaps. In these cases, band splitting induces ferromagnetism (FM) in the g-GaN ML with a total magnetic moment of $1 \mu \mathrm{B}$ per unit cell (Table 1). In order to identify the origin of the magnetic moment, we generated spin-density plots of MLs doped with these group IV impurities, as shown in Fig. 4(a)-4(c), where it can be seen that the magnetic moment is primarily produced by the dopants, whereas the contribution of $\mathrm{N}$ atoms is negligible. The significant $\Delta E$ values of $\mathrm{C}$-, Si-, and Ge-doped systems shown in Table 1 indicate that they can preserve magnetic properties under ambient conditions at room temperature, as $\Delta E$ in the $0.2-0.31 \mathrm{eV}$ range is significantly greater than the thermal energy $(\mathrm{kT}=0.025 \mathrm{eV})$ at room temperature. Thus, the resulting FM is stable at room temperature and can be potentially beneficial for spintronic devices. However, Table 1 indicates that the $\Delta E$ value decreases as the dopant size increases.

To understand the electronic and magnetic properties of group IV-doped systems, the related PDOS is shown in Fig. 3(d)-3(f) for both spin channels, indicating that the PDOS of spin-up and spin-down channels are asymmetric, thus confirming the FM nature of these dopants when introduced to g-GaN. However, the splitting is more pronounced when $\mathrm{Si}$ and $\mathrm{Ge}$ are used as dopants compared to $\mathrm{C}$, which is in agreement 
with the band structure presented in Fig. 2. Fig. 3(d) shows that, in the spin-up configuration of Cdoped GaN, the VBM derives from the $p$ orbitals of $\mathrm{N}$, whereas CBM is a result of both $s$ and $p$ orbitals of $\mathrm{N}$ atoms. For the spin-down channel in C-doped g-GaN, the $\mathrm{N} p$ orbitals contribute to the VBM, and CBM is a result of the $p$ orbitals of C. In the case of Si- and Ge-doped g-GaN MLs, the VBM and CBM originate mainly from the $p$ orbitals of both dopants in both spin channels, as shown in Fig. 3(e) and 3(f). When $\mathrm{C}$ is used as dopant, it interacts more strongly compared to $\mathrm{Si}$ and $\mathrm{Ge}$, as its electronegativity is comparable with the substituted atom $\mathrm{N}$, which is in line with the bond length results, and confirms that the states that do not contribute to bonding hybridize at the CBM. On the other hand, the dopant states of Si and Ge hybridize with the host states at the VBM and CBM in the vicinity of the Fermi level, resulting in the observed room-temperature FM above the thermal energy. The state hybridization between DOS of the g-GaN and the dopants at VBM or CBM in the vicinity of the Fermi level is responsible for the FM of any diluted magnetic semiconductor system, explaining the existence of FM in the group IV-doped g-GaN. Si and Ge dopants tend to lose electrons, which is aligned with the state hybridization at VBM. However, C tends to gain electrons from the $\mathrm{g}-\mathrm{GaN} \mathrm{ML}$, as indicated by the $\delta q$ values reported in Table 1 , explaining the state hybridization near the CBM observed in Fig. 3(d).

For group V dopants, Fig. 2(g)-2(i) show the band structures of $\mathrm{g}-\mathrm{GaN}$ doped with $\mathrm{P}$, As and $\mathrm{Sb}$, respectively. No significant changes are observed in the band structures compared to pure g-GaN ML, as these dopants are isoelectronic atoms and are in the same group as the substituted atom. Therefore, similar to pristine g-GaN, the gGaN ML doped with such elements possesses indirect band gap with nonmagnetic behavior. However, the band gap energy of group V-doped systems is smaller than that of pure $\mathrm{g}-\mathrm{GaN}$, because the bond lengths of these dopants are larger (and the bonds are therefore weaker) than that of $\mathrm{N}$ (as shown in Table 1), as the atomic size of these dopants is larger than $\mathrm{N}$, explaining the smaller band gap of Sb-doped g-GaN compared to that doped with $\mathrm{P}$ and As impurities. The PDOS for P-, As- and Sb-doped g-GaN are shown in Fig. $3(\mathrm{~g})-3(\mathrm{i})$, confirming that $\mathrm{VBM}$ and $\mathrm{CBM}$ are formed by the same cation and anion orbitals originating from pure $\mathrm{g}-\mathrm{GaN}$ ML.

For group VI dopants, the band structures of O-, S- and Se-doped g-GaN ML are presented in Fig. 2(j)-2(1). Interestingly, we find that $\mathrm{ML}$ doped with all group VI elements possesses Fermi level located above the CBM, as shown in Fig. 3(j)-3(1). This indicates that these systems are heavily doped and act as degenerate n-type semiconductors. Upon replacing $\mathrm{N}$ anions with such dopants (which contain one more valence electron than $\mathrm{N}$ does), the ML gains additional electrons, resulting in n-type behavior. Such behavior is attributed to the unpaired electrons that do not participate in the bonding, and therefore form additional energy levels within the g-GaN band gap. Similarly, O incorporated at the N site in the bulk-doped $\mathrm{GaN}$ exhibits n-type behavior ${ }^{52}$.

The PDOS for the O-, S- and Se-doped gGaN ML is shown in Fig. 3(j)-3(1), revealing that these dopants do not contribute significantly to the CBM formation, as such dopants act as extrinsic donors, whereby their electrons do not participate in the crystal bonds. Nonetheless, slight contributions from the dopant states should still exist. We expected the shared electrons in the bond to remain in the vicinity of such dopants for a longer period of time, as the electronegativity of these dopants exceeds that of $\mathrm{N}$, as indicated by their large positive $\delta q$ values (Table 1).

To correct the band gap structures and elucidate the nature of band gaps of the doped systems discussed above, we also presented HSE band gaps along with their PBE values in Table 1, while their band structures are shown in Supporting Information (Fig. S1). As expected, the band gaps of group III-doped systems are widened when the HSE functional is adopted. As shown in Table 1 , the differences between the enhanced band gap energy values and uncorrected values are in the $0.87-1.17 \mathrm{eV}$ range. The group IV-doped g-GaN MLs retain spin-polarized behavior at the HSE level, similar to the PBE, as shown in Fig. 5. However, compared to the 
uncorrected values, the spin-up and spin-down band gap energies produced by the HSE are grater by $1.01-1.40 \mathrm{eV}$ and $1.01-1.48 \mathrm{eV}$, respectively. In addition, the HSE band gaps of group V-doped systems are 0.91-1.04 eV larger than the corresponding PBE values. Similar to the PBE level, the group VI-doped systems show n-type behavior, but their band gaps are widened by 1.12-1.19 eV (see Table 1). The band gap nature of the doped systems is preserved at the HSE level, as their corrected values are roughly proportional to those indicated by the PBE, as shown in Fig. 5(a). It can also be observed that the HSE band gaps of the doped systems follow a hierarchy within the same group, whereby $\mathrm{E}_{\mathrm{g}}(\mathrm{B})>\mathrm{E}_{\mathrm{g}}(\mathrm{Al})>$ $\mathrm{E}_{\mathrm{g}}(\mathrm{In}), \mathrm{E}_{\mathrm{g}(\mathrm{C})}>\mathrm{E}_{\mathrm{g}}(\mathrm{Si})>\mathrm{E}_{\mathrm{g}}(\mathrm{Ge}), \mathrm{E}_{\mathrm{g}}(\mathrm{P})>\mathrm{E}_{\mathrm{g}}(\mathrm{As})>$ $\mathrm{E}_{\mathrm{g}}(\mathrm{Sb}), \mathrm{E}_{\mathrm{g}}(\mathrm{S})>\mathrm{E}_{\mathrm{g}}(\mathrm{Se})>\mathrm{E}_{\mathrm{g}}(\mathrm{O})$. Overall, the difference between the HSE and PBE band gap energy values ranges from 0.87 to $1.48 \mathrm{eV}$, indicating that the doped systems are wide band gap semiconductors and can be used in electronic and optoelectronic device applications.

\section{Electronic and magnetic properties of g-GaN under $\mathrm{N}$-rich conditions}

Here, we discuss the doping effects on the $\mathrm{g}-\mathrm{GaN}$ properties when one $\mathrm{Ga}$ atom is substituted with the selected dopants from group III-VI. The geometric structure of X-doped g-GaN ML at the Ga site is shown in Fig. 1(d), where $X=B, C, A 1$, C, Si, Ge, P, As, Sb, O, S, and Se, under N-rich conditions. The dopant $(\mathrm{X})$ has three nearestneighbor $\mathrm{N}$ atoms.

The calculated bond lengths of $\mathrm{X}-\mathrm{N}$ in doped g-GaN MLs are presented in Table 2. It can be seen that the $\mathrm{X}-\mathrm{N}$ bond length is shorter for doped g-GaN systems when the impurities have smaller radii compared to that of $\mathrm{Ga}-\mathrm{N}$ in pure $\mathrm{g}$ GaN ML, while the large-sized dopants have greater bond lengths (see Table 2). Similar to the Ga-rich environment, no geometric deformation in the planar structure of the ML is induced by doping. In line with the findings reported for Garich conditions, and unlike bulk $\mathrm{GaN}$, the obtained bond lengths $(\mathrm{X}-\mathrm{N})$ are independent of dopant concentration, as the $c$-direction is absent in all 2D materials. The $\mathrm{C}-\mathrm{N}(1.40 \AA)$ and $\mathrm{Al}-\mathrm{N}(1.81 \AA)$ bond lengths calculated in this work are consistent with that in the $\mathrm{N}$-doped graphene ${ }^{53}$ and the 2D AlN sheet ${ }^{34}$, respectively. In the O-doped g-GaN $\mathrm{ML}$, the oxygen atom moves towards one of the nearest-neighbor $\mathrm{N}$ atoms, and it forms the shortest bond (1.20 $\AA$ ) relative to those formed by other dopants, while forming a weak bond of 2.47 $\AA$ A length with the other two neighboring $\mathrm{N}$ atoms. This short bond can be due to the high electron affinities in both $\mathrm{O}$ and $\mathrm{N}$ atoms. It should be noted that we did not find any significant changes in the bond lengths of Ga-X and X-N when the dopants were substituted in different 2D GaN supercells. The band structures of g-GaN MLs in which Ga atoms are replaced by the group III impurities are presented in Fig. 6(a)-6(c). We observe that these band structures exhibit similar behaviors to that of the pure 2D g-GaN ML because the dopants substituting Ga belong to the same group as the cation atom. However, the band gap energy increases and the bond length decreases (i.e., as the dopant atomic size decreases). Thus, the band gap energies of B- and Al-doped g-GaN MLs are higher than that of pure g-GaN, while that obtained for the In-doped ML is lower, as shown in Table 2. The PDOS for the B-, Al-, and Indoped MLs are presented in Fig. 7(a)-7(c), indicating that, as these dopants are isoelectronic atoms, they do not provide a significant contribution to VBM and CBM formation. In addition, the dopant-related states are located away from the Fermi level in the conduction and valence bands. The Bader charge analysis shows that, similar to $\mathrm{Ga}$, all group III dopants lose electrons (as indicated by the $\delta q$ values reported in Table 2) as their electron affinities are smaller than that of the bonded atom (in this case $\mathrm{N}$ ) ${ }^{54}$.

Fig. 6(d)-6(f) show the band structures of g-GaN doped with group IV elements $(\mathrm{C}, \mathrm{Si}$ and Ge), which possess semiconducting properties with n-type conductivity, as these dopants provide donors. When Ga atom is substituted by one of the group IV elements, due to having four valence electrons, such dopants introduce one extra electron, enhancing donor carrier concentrations and inducing n-type behavior, given that the Fermi level shifts above the CBM, as shown in Fig. 6(d)-6(f). The corresponding PDOS of the ML doped with these group IV impurities is shown in 
Fig. 7(d) $-7(\mathrm{f})$. The n-type conductivity arises from the bottom of the conduction band that is derived from the electrons located in non-bonding $s$ and $p$ orbitals of Ga and N. It can also be seen that an additional band originating from $p$ orbitals of IV dopants is formed above the Fermi level. The VBM and CBM of the group IV-doped systems originate from the $\mathrm{N}-s$ and $\mathrm{N}-p$ orbitals, respectively. Akin to group VI-doped systems in the Ga-rich condition, these systems also behave as degenerate semiconductors, as they are heavily doped with donors. It is noteworthy that, unlike g$\mathrm{GaN}$ doped with group IV elements that replace $\mathrm{N}$ and show FM or spin splitting behaviors, these dopants do not exhibit magnetic behavior when dopants replace $\mathrm{Ga}$ atoms. This difference suggests the significance of state hybridization and substitutional site in determining the magnetic behavior of 2D materials. Thus, when group IV elements occupy anion sites, this induces FM and band splitting, whereas no magnetism or band splitting is observed when the same dopants replace cations in $\mathrm{g}-\mathrm{GaN}$ MLs.

For group $\mathrm{V}$ elements, the systems doped by substituting $\mathrm{Ga}$ with $\mathrm{P}$, As and $\mathrm{Sb}$ behave as a narrow indirect band gap semiconductor, as shown in Fig. 6(g)-6(i) and Table 2. The V dopants significantly reduce the band gap energy compared to other dopants. In this case, $\mathrm{N}$ are bonded with dopants belong to the same group, affecting the polarity of the X-N bond, which may affect the band structure. Furthermore, P-doped g$\mathrm{GaN}$ possesses the lowest band gap energy $(0.29$ $\mathrm{eV}$ ) and its Fermi level shifts much closer to the CBM compared to the other dopants, although the bond length is shorter than that of other group $\mathrm{V}$ dopant $-\mathrm{N}$ pairs. The PDOS shown in Fig. $7(\mathrm{~g})-7(\mathrm{i})$ indicates that the CBM is dominated by the $p$ states from $\mathrm{P}$, As and $\mathrm{Sb}$ atoms, while the $p$ orbitals of $\mathrm{N}$ contribute equally to the CBM in Pdoped ML, which can be due to their short bonds, leading to relatively n-type conductivity. The VBMs of group V-doped systems are derived from $p$ orbitals of both $\mathrm{N}$ and the dopant atoms.

Finally, the band structures of the group VI dopants that form O- S- and Se-doped g-GaN ML are shown in Fig. 6(j)-6(l). Interestingly, when occupying $\mathrm{Ga}$ sites, these dopants induced magnetic behavior, as well as spin-up and spindown band splitting, as indicated by the asymmetric PDOS. The total magnetic moments (1-3 $\mu_{\mathrm{B}}$ per unit cell) are significant, as shown in Table 2 . The spin density plot provided in Fig. 4 shows that most contributions to the magnetic moment can be attributed to polarized states of such dopants hybridizing with the surrounding atoms. The MM corresponding to the O-doped system is much greater $(3 \mu \mathrm{B})$ than that of Se- and S-doped ML $(1 \mu \mathrm{B})$ because the two nearest $\mathrm{N}$ neighbors form a weak bond with $\mathrm{O}$. Thus, the unpaired electrons from the $\mathrm{N}$ atoms (corresponding to the dumbbell shape in Fig. 4 (d)) contribute to such a large magnetic moment. The significant energy difference between the spinpolarized and unpolarized configurations $(\Delta E=$ $0.14-0.39 \mathrm{eV}$ ) suggests that the doped systems exhibit FM at the ground state at room temperature. Similar to the magnetic systems studied under Ga-rich conditions, the $\Delta E$ value decreases as the dopant atomic size increases (Table 2). The $\delta q$ values reported in Table 2 show that, when replacing $\mathrm{Ga}, \mathrm{O}$ atom tends to gain electrons relative to $\mathrm{N}$, whereas other substitutional dopants tend to lose electrons when replacing Ga. This suggests that the electronegativity of the $\mathrm{O}$ atoms is greater than that of the bonded atom $\mathrm{N}^{55}$.

Similar to the work carried out under Garich conditions, we also calculated the band gaps of doped systems using HSE06 and the findings are presented in Table 2. Their corresponding band structures are provided in Fig. S5, Supporting Information. We found that band gaps of all doped systems (except those doped with group $\mathrm{V}$ elements) are significantly modified, with the correction in the band gap energy exceeding 1 $\mathrm{eV}$. This discrepancy is due to the smaller band gaps characterizing group V-doped systems at the PBE level compared to other doped systems. Upon inclusion of the HSE functional, band gaps of these systems are widened by $0.23-0.60 \mathrm{eV}$, as shown in Table 2. Moreover, at the HSE level, systems doped with group IV and VI elements exhibit n-type behavior and spin-polarized behavior, respectively (see Fig. S5 in Supporting Information), which is in line with the PBE 
calculations. However, HSE calculations induce a $0.58 \mathrm{eV}$ and $1.48 \mathrm{eV}$ increase in the corrected spinup and spin-down band gap energies, respectively, compared to the uncorrected values. Akin to the Ga-rich conditions, the HSE band gaps are proportional to the relevant $\mathrm{PBE}$ band gaps under N-rich conditions, as shown in Fig. 5(b). It is also evident from Fig. 5(b) and Table 2 that the band gaps calculated using the HSE functional show the following hierarchy within the groups: $\mathrm{E}_{\mathrm{g}}(\mathrm{B})>$ $\mathrm{E}_{\mathrm{g}}(\mathrm{Al})>\mathrm{E}_{\mathrm{g}}(\mathrm{In}) ; \mathrm{E}_{\mathrm{g}}(\mathrm{Si})>\mathrm{E}_{\mathrm{g}}(\mathrm{C})>\mathrm{E}_{\mathrm{g}}(\mathrm{Ge}) ; \mathrm{E}_{\mathrm{g}}(\mathrm{Sb})>$ $\mathrm{E}_{\mathrm{g}}(\mathrm{As})>\mathrm{E}_{\mathrm{g}}(\mathrm{P}) ; \mathrm{E}_{\mathrm{g}}(\mathrm{O})>\mathrm{E}_{\mathrm{g}}(\mathrm{Se})>\mathrm{E}_{\mathrm{g}}(\mathrm{S})$. Hence, the HSE calculations confirm the interesting behavior the studied systems exhibited at the PBE level, while predicting significantly higher band gap energies. These findings would be beneficial when considering these doped materials for potential practical applications.

\section{Concentration dependence on the band gap of doped g-GaN}

Here, we examine the band gap behavior of doped g-GaN ML as a function of dopant concentration. The assessment includes all dopants considered in this study and is conducted using the PBE functional because it is computationally very expensive to calculate the HSE band gaps of the doped systems for all concentrations of interest for this investigation as we found that HSE band gap structures show trends similar to that estimated by PBE calculations although the estimated energy gap is underestimated by PBE.

The band gap behavior of doped systems as a function of dopant concentration, whereby the dopants occupy $\mathrm{N}$ sites under Ga-rich conditions, is illustrated in Fig. 8. It can be seen in Fig. 8(a) that the band gap decreases as the group III dopant concentration increases, given that the band gap values $(\leq 1 \mathrm{eV})$ are smaller than that obtained for pristine g-GaN ML. When the systems doped with group III elements are examined, the lowest band gap value $(0.09 \mathrm{eV})$ is obtained for the In-doped g-GaN ML (at 5.5\% concentration) due to its long bonds, as shown in Fig. S2 in Supporting Information. It is worth noting that the g-GaN MLs doped with group III atoms exhibit direct band gap behavior at the $1.38 \%$ concentration
(Fig. S4 in Supporting Information). However, higher concentrations result in the emergence of both direct and indirect band gap, depending on the dopant type and its concentration. For example, when g-GaN ML is doped with $\mathrm{B}$ at $3.125 \%, \mathrm{Al}$ at $5.5 \%$, and $\mathrm{In}$ at $3.125 \%$ and $2 \%$, its band gap becomes direct (Fig. S2 and S3 in Supporting Information). Such change is due to the bond polarity, as more polar bonds correspond to a more direct band gap structure ${ }^{56}$. As discussed above, g-GaN doped with group IV elements possesses both spin-up and spin-down band gaps. We found that the band gaps of the C-doped system are weakly dependent on dopant concentration, while the band gap energies of Siand Ge-doped structures decrease as the dopant concentration increases, as shown in Fig. 8(b). Fig. 8(c) illustrates the band gap energy of g-GaN ML doped with group $\mathrm{V}$ elements, replacing $\mathrm{N}$. Similar to the findings reported for the system doped by group III impurities, the band gap of P-, As- and Sb-doped GaN decreases as the dopant concentration increases. The band gaps of group V-doped g-GaN MLs are greater than those of the group III-doped MLs, but are smaller than those pertaining to pure ML, as shown in Table 1 . Moreover, all group V dopants induce direct band gap behavior at $2 \%$ dopant concentration. However, P- and As-doped MLs also have direct band gaps at higher dopant concentrations (up to $5.5 \%$ ). On the other hand, the group VI dopant concentration dependence under Ga-rich conditions is not shown here, as such doped systems exhibit n-type conductivity at all dopant concentrations considered in this study. The above discussion suggests that substituting $\mathrm{N}$ by such dopants plays a significant role in the electronic and magnetic properties of 2D GaN.

The band gap behavior of the doped g-GaN MLs as a function of dopant concentration, when the dopants occupy Ga sites under N-rich conditions, is shown Fig. 9. It can be seen that the band gap of the B- and Al-doped MLs increases and that of In-doped ML decreases as the dopant concentration increases. Interestingly, g-GaN MLs doped with group III elements have direct band gaps at all dopant concentrations in the $1.38-5.5 \%$ range (Fig. S8 and S6, Supporting 
Information). This direct band gap behavior is due to the bond polarity that remains unchanged within the examined concentration range. In addition, MLs doped with group IV elements exhibit n-type behavior at all dopant concentrations. However, the band gap of group V-doped MLs decreases as dopant concentration increases, as shown in Fig. 9(b). The obtained band gap becomes direct when As and $\mathrm{Sb}$ concentrations reach $5.5 \%$. As mentioned above, doping systems with group VI atoms introduces spin splitting. In contrast to its behavior in Ga-rich environment, the P-doped ML shows indirect band gap at all concentrations in $\mathrm{N}$ rich environment. As shown in Fig. 9(c), spin-up band gap increases and spin-down band gap decreases with increasing dopant concentration. MLs doped with group III and V elements exhibit an indirect band gap at $2 \%$ dopant concentration (Fig. S7 in Supporting Information). Worth mentioning that band gap structure depends on the distribution of the dopants in a GaN monolayer ${ }^{57,58}$. Therefore, we examined the distribution of two dopants in a $6 \times 6 \mathrm{~g}-\mathrm{GaN}$ supercell (Fig. S13), as discussed in Section S7. In this case, we study the most stable dopants $(\mathrm{O}$ and $\mathrm{Al}$ ) under both $\mathrm{Ga}-$ and $\mathrm{N}$ - rich conditions atoms that are substituted $\mathrm{N}$ or Ga, respectively. Fig. S14 shows the band structures of most stable configurations in Section S7 in the supporting information.

\section{Stability}

Substitution energies: In order to establish the thermodynamic stability of any doped system, it is essential to have an in-depth understanding of the formation or substitutional energy. We calculated the substitution energies $\left(E_{s}\right)$ of doped systems under both Ga-rich and N-rich doping environments by using the following expression: ${ }^{59,60}$

$E_{s}=E(G a N+X)-E(G a N)-\mu_{X}+\mu_{G a} / \mu_{N}$

where $E(G a N+X)$ is the total energy of the g-GaN ML doped with a dopant $\mathrm{X}, E(G a N)$ is the total energy of pure $\mathrm{GaN}$ supercell corresponding to the doped system, $\mu_{X}$ denotes the chemical potential of the dopant, and $\mu_{G a} / \mu_{N}$ represents the chemical potential of one $\mathrm{Ga}$ or $\mathrm{N}$ atom that is substituted. The chemical potentials of all dopants (except $\mathrm{O}$ ) are calculated from total energies of their bulk structures. For the $\mathrm{O}$ atom, it is instead derived from the total energy per atom of the $\mathrm{O}_{2}$ molecule. According to the results reported in pertinent literature ${ }^{52}, \mu_{G a}=\mu_{G a}$ (bulk) holds under Ga-rich conditions, while in N-rich conditions it is calculated as $\mu_{N}=0.5^{*} \mu_{N 2}$, where $\mu_{N 2}$ denotes total energy per atom in $\mathrm{N}_{2}$ molecule. As the equilibrium is given by:

$$
\mu_{\text {GaN }}=\mu_{G a+} \mu_{N}
$$

where, $\mu_{\text {GaN }}$ is the total energy of a primitive $\mathrm{GaN}$ cell with two atoms. From Eq. (2), the chemical potentials of $\mathrm{Ga}$ and $\mathrm{N}$ in the lower bounds are defined as: $\mu_{G a}=\mu_{G a N}-\mu_{N}$ and $\mu_{N}=\mu_{G a N}-\mu_{G a}$.

The calculated $\mathrm{E}_{\mathrm{s}}$ for $\mathrm{Ga}-$ and N-rich doping environments are presented in Table 1 and Fig. S9, and Table 2 and Fig. S10, respectively. Under Ga-rich conditions, only O-doped $\mathrm{GaN}$ has negative substitutional energy (see Fig. S9 in Supporting Information), indicating that all other dopant substitutions require energy. In addition, across all groups, dopants of smaller atomic sizes occupying $\mathrm{N}$ sites have lower substitution energies. On the other hand, B-, Al- and Si-doped systems have negative substitution energies, which contributes to high stability under N-rich conditions (see Fig. S10). This stability is attributed to the short and strong $\mathrm{B}-\mathrm{N}, \mathrm{Al}-\mathrm{N}$, and $\mathrm{Si}-\mathrm{N}$ bonds. Moreover, $\mathrm{Al}, \mathrm{B}$ and $\mathrm{Si}$ atoms preferentially substitute $\mathrm{Ga}$, leading to high solubility, which can be due to the atomic radius of these dopants occupying Ga sites.

When the $E_{s}$ of the doped systems are examined in relation to dopant concertation, the findings indicate that, under both Ga- and N-rich conditions, the formation energies of MLs doped with all the impurities are independent of concentration, as shown in Fig. S9 and Fig. S10 in the Supporting Information. Based on the findings reported in this work, we can surmise that the Odoped system is the most stable under Ga-rich conditions because the atomic size of the dopant is roughly equal to that of the anion $(\mathrm{N})$ it replaces, leading to stable bonds between them. Our predictions of n-type behavior in $\mathrm{O}-, \mathrm{C}-$ and $\mathrm{Si}-$ 
doped systems are in good agreement with that observed for the bulk doped $\mathrm{GaN}^{52}$. In sum, $\mathrm{O}$ and $\mathrm{Al}$ atoms are the most suitable candidates for doping g-GaN in Ga- and N-rich environments, respectively. Similar to doped bulk systems, we expect that doped g-GaN structures can be realized in the future, allowing them to be utilized in nanoelectronic device applications.

Effect of temperature on stability: To examine the effect of temperature on the most preferred doping configurations-O-doped $\mathrm{GaN}$ in Ga-rich conditions and Al-doped GaN in N-rich conditions - we performed MD simulations for the doped configurations at various temperatures. The plots shown in Fig. 10 present the total energy fluctuations of the doped configurations as a function of simulated time (ps) at various temperatures $(300 \mathrm{~K}, 500 \mathrm{~K}$ and $1000 \mathrm{~K})$. It can be clearly seen that the energy fluctuations remain in a relatively narrow range $(<0.08 \mathrm{eV})$ even at the highest temperature of $1000 \mathrm{~K}$. The simulated geometries for both doped configurations at 1000 $\mathrm{K}$ are presented in Fig. S11, Supporting Information. At the high temperatures considered in this investigation, although atoms slightly shift from their equilibrium positions, there is no planar deformation. These findings indicate that the doped configurations discussed here exhibit high stability, indicating high melting points.

\section{CONCLUSION}

In this work, we have presented the electronic and magnetic properties of substitutionally doped 2D GaN MLs using the DFT approach and MD simulations. We also employed a computationally expensive HSE approach in order to verify interesting behaviors of certain doping systems. We considered the dopants from groups III-VI and have varied their concentration in the $1.38-5.5 \%$ range. We included the dopants interacting with the monolayer in Ga- and N-rich doping environments. Our electronic structure calculations suggest that group III and V dopants retain the semiconducting property of $2 \mathrm{D} \mathrm{GaN}$ in both environments. The dopants also transform the ML into a direct band gap semiconductor, based on the dopant type concentration and substitutional site. Group IV- and group VI-doped systems show either nonmagnetic behavior with ntype conductivity, or are magnetic with spinpolarized behavior and finite magnetic moments. Our substitutional energy calculations suggest that 2D GaN is preferentially doped with the elements of a smaller atomic size relative to that of the replaced atom. Thus, oxygen is the most suitable dopant in the Ga-rich environment, whereas B, Al, and $\mathrm{Si}$ are preferred for the N-rich conditions. Finally, the band gap values corrected using the HSE functional are $0.23-1.48 \mathrm{eV}$ greater than the corresponding PBE values. Therefore, our predictions could assist in tuning the electronic and magnetic properties of $2 \mathrm{D} \mathrm{GaN}$ in different doping environments, which would be highly beneficial for material growers. Owing to the properties demonstrated in this work, we are confident that the potential of $2 \mathrm{D} \mathrm{GaN}$ for use in diverse applications, such as fabrication of optoelectronic and spintronic devices, has been confirmed.

\section{ASSOCIATED CONTENT}

\section{Supporting information}

PBE and HSE band structures of X-doped g-GaN under both Ga-and N-rich conditions for different dopant concentrations. Geometrical structures of g-GaN with different dopant concentrations. Band structures of $\mathrm{GaN}$ doped with two $\mathrm{Al}$ and $\mathrm{O}$ atoms. Substitution energies as a function of $\mathrm{X}$ concentration under $\mathrm{Ga}$ - and $\mathrm{N}$-rich conditions

\section{AUTHOR INFORMATION}

\section{Corresponding Author}

Email: iman.roqan@kaust.edu.sa

\section{Note}

The authors declare no competing conflict of interest.

\section{ACKNOWLEDGEMENTS}


NA and IR acknowledge the computing support provided by Shaheen II at the King Abdullah University of Science and Technology (KAUST). Authors thanks KAUST for the finance support.

\section{References}

(1) Muhammed, M. M.; Alwadai, N.; Lopatin, S.; Kuramata, A.; Roqan, I. S. High-Efficiency InGaN/GaN Quantum WellBased Vertical Light-Emitting Diodes Fabricated on $\beta-\mathrm{Ga}_{2} \mathrm{O}_{3}$ Substrate. ACS Appl. Mater. Interfaces 2017, 9, $34057-$ 34063.

(2) Roqan, I. S.; O’Donnell, K. P.; Martin, R. W.; Edwards, P. R.; Song, S. F.; Vantomme, A.; Lorenz, K.; Alves, E.; Boćkowski, M. Identification of the Prime Optical Center in GaN : Eu 3 +. Phys. Rev. B 2010, 81, 085209.

(3) Ajia, I. A.; Edwards, P. R.; Pak, Y.; Belekov, E.; Roldan, M. A.; Wei, N.; Liu, Z.; Martin, R. W.; Roqan, I. S. Generated Carrier Dynamics in V-Pit-Enhanced InGaN/GaN Light-Emitting Diode. ACS Photonics 2018, 5, 820-826.

(4) Tsao, J. Y.; Chowdhury, S.; Hollis, M. A.; Jena, D.; Johnson, N. M.; Jones, K. A.; Kaplar, R. J.; Rajan, S.; Van de Walle, C. G.; Bellotti, E.; et al. Ultrawide-Bandgap Semiconductors: Research Opportunities and Challenges. Adv. Electron. Mater. 2018, 4, 1600501.

(5) Zhang, Z.; Schwingenschlögl, U.; Roqan, I. S. Vacancy Complexes Induce Long-Range Ferromagnetism in GaN. $J$. Appl. Phys. 2014, 116, 183905.

(6) Zhu, D.; Wallis, D. J.; Humphreys, C. J. Prospects of III-Nitride Optoelectronics Grown on Si. Reports Prog. Phys. 2013, 76, 106501.

(7) Kecik, D.; Onen, A.; Konuk, M.; Gürbüz, E.; Ersan, F.; Cahangirov, S.; Aktürk, E.; Durgun, E.; Ciraci, S. Fundamentals, Progress, and Future Directions of Nitride-Based Semiconductors and Their Composites in TwoDimensional Limit: A First-Principles Perspective to Recent Synthesis. Appl. Phys. Rev. 2018, 5, 011105.

(8) Zhang, H.; Wu, D.; Tang, Q.; Liu, L.; Zhou, Z. ZnO-GaN Heterostructured Nanosheets for Solar Energy Harvesting: Computational Studies Based on Hybrid Density Functional Theory. J. Mater. Chem. A 2013, 1, 2231-2237.

(9) Pashartis, C.; Rubel, O. Alloying Strategy for Two-Dimensional GaN Optical Emitters. Phys. Rev. B 2017, $96,155209$.

(10) Wang, V.; Wu, Z. Q.; Kawazoe, Y.; Geng, W. T. Tunable Band Gaps of In X Ga 1- X N Alloys: From Bulk to TwoDimensional Limit. J. Phys. Chem. C 2018, 122, 6930-6942.

(11) Novoselov, K.; Geim, A.; Morozov, S.; Jiang, D.; Zhang, Y.; Dubonos, S.; Grigorieva, I.; Firsov, A. Electric Field Effect in Atomically Thin Carbon Films. Science 2004, 306, 666-669.

(12) Geim, A. K.; Novoselov, K. S. The Rise of Graphene. Nat. Mater. 2007, 6, 183-191.

(13) Katsnelson, M. I. Graphene: Carbon in Two Dimensions. Mater. today 2007, 10, 20-27.

(14) Castro Neto, A. H.; Guinea, F.; Peres, N. M. R.; Novoselov, K. S.; Geim, A. K. The Electronic Properties of Graphene. Rev. Mod. Phys. 2009, 81, 109-162.

(15) Coleman, J. N.; Lotya, M.; O’Neill, A.; Bergin, S. D.; King, P. J.; Khan, U.; Young, K.; Gaucher, A.; De, S.; Smith, R. J.; et al. Two-Dimensional Nanosheets Produced by Liquid Exfoliation of Layered Materials. Science 2011, 331, 568-571.

(16) Zhang, B. Y.; Liu, T.; Meng, B.; Li, X.; Liang, G.; Hu, X.; Wang, Q. J. Broadband High Photoresponse from Pure Monolayer Graphene Photodetector. Nat. Commun. 2013, 4, 1811.

(17) Mannix, A. J.; Zhou, X.-F.; Kiraly, B.; Wood, J. D.; Alducin, D.; Myers, B. D.; Liu, X.; Fisher, B. L.; Santiago, U.; Guest, J. R.; et al. Synthesis of Borophenes: Anisotropic, Two-Dimensional Boron Polymorphs. Science 2015, 350, $1513-1516$.

(18) Liu, H.; Neal, A. T.; Zhu, Z.; Luo, Z.; Xu, X.; Tománek, D.; Ye, P. D. Phosphorene: An Unexplored 2D Semiconductor with a High Hole Mobility. ACS Nano 2014, 8, 4033-4041.

(19) Ci, L.; Song, L.; Jin, C.; Jariwala, D.; Wu, D.; Li, Y.; Srivastava, A.; Wang, Z. F.; Storr, K.; Balicas, L.; et al. Atomic Layers of Hybridized Boron Nitride and Graphene Domains. Nat. Mater. 2010, 9, 430-435.

(20) Wang, Q. H.; Kalantar-Zadeh, K.; Kis, A.; Coleman, J. N.; Strano, M. S. Electronics and Optoelectronics of TwoDimensional Transition Metal Dichalcogenides. Nat. Nanotechnol. 2012, 7, 699-712.

(21) Naguib, M.; Mashtalir, O.; Carle, J.; Presser, V.; Lu, J.; Hultman, L.; Gogotsi, Y.; Barsoum, M. W. Two-Dimensional Transition Metal Carbides. ACS Nano 2012, 6, 1322-1331.

(22) Vogt, P.; De Padova, P.; Quaresima, C.; Avila, J.; Frantzeskakis, E.; Asensio, M. C.; Resta, A.; Ealet, B.; Le Lay, G. Silicene: Compelling Experimental Evidence for Graphenelike Two-Dimensional Silicon. Phys. Rev. Lett. 2012, 108, 155501.

(23) Geim, A. K.; Grigorieva, I. V. Van Der Waals Heterostructures. Nature 2013, 499, 419-425.

(24) Al Balushi, Z. Y.; Wang, K.; Ghosh, R. K.; Vilá, R. A.; Eichfeld, S. M.; Caldwell, J. D.; Qin, X.; Lin, Y.-C.; DeSario, P. A.; Stone, G.; et al. Two-Dimensional Gallium Nitride Realized via Graphene Encapsulation. Nat. Mater. 2016, 15, 
1166-1171.

(25) Dávila, M. E.; Xian, L.; Cahangirov, S.; Rubio, A.; Le Lay, G. Germanene: A Novel Two-Dimensional Germanium Allotrope Akin to Graphene and Silicene. New J. Phys. 2014, 16, 095002.

(26) Şahin, H.; Cahangirov, S.; Topsakal, M.; Bekaroglu, E.; Akturk, E.; Senger, R. T.; Ciraci, S. Monolayer Honeycomb Structures of Group-IV Elements and III-V Binary Compounds: First-Principles Calculations. Phys. Rev. B 2009, 80, 155453.

(27) Özçelik, V. O.; Aktürk, O. Ü.; Durgun, E.; Ciraci, S. Prediction of a Two-Dimensional Crystalline Structure of Nitrogen Atoms. Phys. Rev. B 2015, 92, 125420.

(28) Chen, Q.; Hu, H.; Chen, X.; Wang, J. Tailoring Band Gap in GaN Sheet by Chemical Modification and Electric Field: Ab Initio Calculations. Appl. Phys. Lett. 2011, 98, 053102.

(29) Guo, J.; Zhou, Z.; Wang, T.; Lu, Z.; Yang, Z.; Liu, C. Electronic Structure and Optical Properties for Blue Phosphorene/graphene-like GaN van Der Waals Heterostructures. Curr. Appl. Phys. 2017, 17.

(30) Mu, Y. Chemical Functionalization of GaN Monolayer by Adatom Adsorption. J. Phys. Chem. C 2015, 119, 2091120916.

(31) Yong, Y.; Cui, H.; Zhou, Q.; Su, X.; Kuang, Y.; Li, X. Adsorption of Gas Molecules on a Graphitic GaN Sheet and Its Implications for Molecule Sensors. RSC Adv. 2017, 7, 51027-51035.

(32) Kadioglu, Y.; Ersan, F.; Kecik, D.; Aktürk, O. Ü.; Aktürk, E.; Ciraci, S. Chemical and Substitutional Doping, and Anti-Site and Vacancy Formation in Monolayer AlN and GaN. Phys. Chem. Chem. Phys. 2018, 20, $16077-16091$.

(33) Meng, B.; Xiao, W. Z.; Wang, L. L.; Yue, L.; Zhang, S.; Zhang, H. Y. Magnetic Properties in a IIIA-Nitride Monolayer Doped with Cu: A Density Functional Theory Investigation. RSC Adv. 2015, 5, 82357-82362.

(34) Peng, Y.; Xia, C.; Zhang, H.; Wang, T.; Wei, S.; Jia, Y. Tunable Electronic Structures of P -Type Mg Doping in AlN Nanosheet. J. Appl. Phys. 2014, 116, 044306.

(35) Amorim, R. G.; Zhong, X.; Mukhopadhyay, S.; Pandey, R.; Rocha, A. R.; Karna, S. P. Strain- and Electric FieldInduced Band Gap Modulation in Nitride Nanomembranes. J. Phys. Condens. Matter 2013, 25, 195801.

(36) Zhao, Q.; Xiong, Z.; Qin, Z.; Chen, L.; Wu, N.; Li, X. Tuning Magnetism of Monolayer GaN by Vacancy and Nonmagnetic Chemical Doping. J. Phys. Chem. Solids 2016, 91, 1-6.

(37) González, R.; López-Pérez, W.; González-García, Á.; Moreno-Armenta, M. G.; González-Hernández, R. Vacancy Charged Defects in Two-Dimensional GaN. Appl. Surf. Sci. 2018, 433, 1049-1055.

(38) Cai, Y.; Zhang, G.; Zhang, Y.-W. Layer-Dependent Band Alignment and Work Function of Few-Layer Phosphorene. Sci. Rep. 2015, 4, 6677.

(39) Bianco, E.; Butler, S.; Jiang, S.; Restrepo, O. D.; Windl, W.; Goldberger, J. E. Stability and Exfoliation of Germanane: A Germanium Graphane Analogue. ACS Nano 2013, 7, 4414-4421.

(40) Onat, B.; Hallioglu, L.; İpek, S.; Durgun, E. Tuning Electronic Properties of Monolayer Hexagonal Boron Phosphide with Group III-IV-V Dopants. J. Phys. Chem. C 2017, 121, 4583-4592.

(41) Kresse, G.; Furthmüller, J. Efficient Iterative Schemes for Ab Initio Total-Energy Calculations Using a Plane-Wave Basis Set. Phys. Rev. B 1996, 54, 11169-11186.

(42) Kresse, G.; Furthmüller, J. Efficiency of $\backslash$ emph $\{$ ab Initio $\}$ Total Energy Calculations for Metals and Semiconductors Using a Plane-Wave Basis Set. Comput. Mater. Sci. 1996, 6, 15-50.

(43) Perdew, J. P.; Burke, K.; Ernzerhof, M. Generalized Gradient Approximation Made Simple. Phys. Rev. Lett. 1996, 77, 3865-3868.

(44) Henkelman, G.; Arnaldsson, A.; Jónsson, H. A Fast and Robust Algorithm for BaderDecomposition of Charge Density. Comput. Mater. Sci. 2006, 36, 354.

(45) Paier, J.; Marsman, M.; Hummer, K.; Kresse, G.; Gerber, I. C.; Ángyán, J. G. Screened Hybrid Density Functionals Applied to Solids. J. Chem. Phys. 2006, 124, 154709.

(46) Hoover, W. G. Canonical Dynamics: Equilibrium Phase-Space Distributions. Phys. Rev. A $1985,31,1695-1697$.

(47) Xia, C.; Peng, Y.; Wei, S.; Jia, Y. The Feasibility of Tunable P-Type Mg Doping in a GaN Monolayer Nanosheet. Acta Mater. 2013, 61, 7720-7725.

(48) Tang, W.; Sun, M.; Yu, J.; Chou, J.-P. Magnetism in Non-Metal Atoms Adsorbed Graphene-like Gallium Nitride Monolayers. Appl. Surf. Sci. 2018, 427, 609-612.

(49) Özçelik, V. O.; Azadani, J. G.; Yang, C.; Koester, S. J.; Low, T. Band Alignment of Two-Dimensional Semiconductors for Designing Heterostructures with Momentum Space Matching. Phys. Rev. B 2016, 94, 035125.

(50) Fan, S. W.; Huang, X. N.; Yao, K. L. Boron Doped GaN and InN: Potential Candidates for Spintronics. J. Appl. Phys. 2017, 121, 073905.

(51) He, C.-N.; Huang, W.-Q.; Xu, L.; Yang, Y.-C.; Zhou, B.-X.; Huang, G.-F.; Peng, P.; Liu, W.-M. Tuning near-Gap Electronic Structure, Interface Charge Transfer and Visible Light Response of Hybrid Doped Graphene and Ag3PO4 Composite: Dopant Effects. Sci. Rep. 2016, 6, 22267.

(52) Van de Walle, C. G.; Neugebauer, J. First-Principles Calculations for Defects and Impurities: Applications to IIINitrides. J. Appl. Phys. 2004, 95, 3851-3879. 
(53) Dai, J.; Yuan, J.; Giannozzi, P. Gas Adsorption on Graphene Doped with B, N, Al, and S: A Theoretical Study. Appl. Phys. Lett. 2009, 95, 232105.

(54) Myers, R. T. The Periodicity of Electron Affinity. J. Chem. Educ. 1990, 67, 307.

(55) Little, E. J.; Jones, M. M. A Complete Table of Electronegativities. J. Chem. Educ. 1960, $37,231$.

(56) Harrison, W. Electronic Structure and the Properties of Solids: The Physics of the Chemical Bond; 2012.

(57) Guo, H.; Zhao, Y.; Lu, N.; Kan, E.; Zeng, X. C.; Wu, X.; Yang, J. Tunable Magnetism in a Nonmetal-Substituted ZnO Monolayer: A First-Principles Study. J. Phys. Chem. C 2012, 116, 11336-11342.

(58) Huang, H.; Li, Z.; She, J.; Wang, W. Oxygen Density Dependent Band Gap of Reduced Graphene Oxide. J. Appl. Phys. 2012, 111, 054317.

(59) Jin, M.; Li, Z.; Huang, F.; Xia, Y.; Ji, X.; Wang, W. Critical Conditions for the Formation of P-Type ZnO with Li Doping. RSC Adv. 2018, 8, 30868-30874.

(60) Horzum, S.; Çakır, D.; Suh, J.; Tongay, S.; Huang, Y.-S.; Ho, C.-H.; Wu, J.; Sahin, H.; Peeters, F. M. Formation and Stability of Point Defects in Monolayer Rhenium Disulfide. Phys. Rev. B 2014, 89, 155433. 


\section{Tables and Figures}

Table 1. The dopant atom (X), PBE and HSE (parentheses) band gaps $\left(\mathrm{E}_{\mathrm{g}}\right)$ of the X-doped systems, bond lengths between the dopant and the $\mathrm{N}$ atom $(\mathrm{X}-\mathrm{N})$, lattice constant $(a)$, total magnetic moment of the $\mathrm{X}$ doped system $(\mathrm{MM})$, substitution energy $\left(\mathrm{E}_{\mathrm{s}}\right)$, energy difference between magnetic and nonmagnetic configurations $(\Delta \mathrm{E})$, and the dopant charge accumulated or lost $(\delta q)$.

\begin{tabular}{llllllll}
\hline $\mathrm{X}$ & $\mathrm{E}(\mathrm{eV})$ & $\begin{array}{l}\mathrm{X}- \\
\mathrm{Ga} \\
(\AA)\end{array}$ & $\begin{array}{l}\mathrm{a} \\
(\AA)\end{array}$ & $\begin{array}{l}\mathrm{M} \\
\left(\mu_{\mathrm{B}}\right)\end{array}$ & $\begin{array}{l}\mathrm{E}_{\mathrm{s}} \\
(\mathrm{eV})\end{array}$ & $\begin{array}{l}\Delta \mathrm{E} \\
(\mathrm{eV})\end{array}$ & $\begin{array}{l}\delta \mathrm{q} \\
(e)\end{array}$ \\
\hline $\mathrm{B}$ & $0.86(1.90)$ & 2.02 & 13.13 & 0 & 3.34 & - & +0.09 \\
$\mathrm{Al}$ & $0.87(1.74)$ & 2.30 & 13.28 & 0 & 4.02 & - & -1.08 \\
$\mathrm{In}$ & $0.58(1.75)$ & 2.38 & 13.33 & 0 & 4.69 & - & -0.10 \\
$\mathrm{C}$ & $\mathrm{Up} \mathrm{2.00(3.40)}$ & 1.94 & 13.06 & 1 & 1.81 & 0.31 & +0.90 \\
& $\mathrm{Dn} \mathrm{1.08(2.56)}$ & & & & & & \\
$\mathrm{Si}$ & $\mathrm{Up} \mathrm{1.23(2.25)}$ & 2.23 & 13.23 & 1 & 3.26 & 0.23 & -0.77 \\
& $\mathrm{Dn} 1.33(2.30)$ & & & & & & \\
$\mathrm{Ge}$ & $\mathrm{Up} \mathrm{1.03(2.04)}$ & 2.26 & 13.24 & 1 & 3.10 & 0.20 & -0.29 \\
& $\mathrm{Dn} \mathrm{1.19(2.20)}$ & & & & & & \\
$\mathrm{P}$ & $1.47(2.51)$ & 2.18 & 13.18 & 0 & 0.86 & - & +0.35 \\
$\mathrm{As}$ & $1.20(2.17)$ & 2.24 & 13.21 & 0 & 1.36 & - & +0.10 \\
$\mathrm{Sb}$ & $0.80(1.71)$ & 2.37 & 13.28 & 0 & 3.08 & - & -0.14 \\
$\mathrm{O}$ & $1.60(2.79)$ & 1.94 & 13.07 & 0 & -1.82 & - & +1.02 \\
$\mathrm{~S}$ & $1.75(2.89)$ & 2.25 & 13.22 & 0 & 0.83 & - & +0.44 \\
$\mathrm{Se}$ & $1.72(2.84)$ & 2.33 & 13.26 & 0 & 1.34 & - & +0.01 \\
\hline & & & & & & & \\
\hline
\end{tabular}


Table 2. The dopant atom (X), PBE and HSE (parentheses) band gaps $\left(\mathrm{E}_{\mathrm{g}}\right)$ of the X-doped systems, bond lengths between the dopant and the $\mathrm{Ga}$ atom $(\mathrm{X}-\mathrm{Ga})$, lattice constant $(a)$, total magnetic moment $(\mathrm{MM})$ of the X-doped system, substitution energy $\left(E_{s}\right)$, the energy difference between magnetic and nonmagnetic configurations $(\Delta \mathrm{E})$, and the dopant charge gain or loss $(\delta \mathrm{q})$.

\begin{tabular}{|c|c|c|c|c|c|c|c|}
\hline $\mathrm{X}$ & $\mathrm{E}_{\mathrm{g}}(\mathrm{eV})$ & $\begin{array}{l}\mathrm{X}-\mathrm{N} \\
(\AA)\end{array}$ & $\begin{array}{l}\text { a } \\
(\AA)\end{array}$ & $\begin{array}{l}\mathrm{M} \\
\mathrm{M} \\
\left(\mu_{\mathrm{B}}\right)\end{array}$ & $\begin{array}{l}E_{s} \\
(e V)\end{array}$ & $\begin{array}{l}\Delta \mathrm{E} \\
(\mathrm{eV})\end{array}$ & $\begin{array}{l}\delta \mathrm{q} \\
(e)\end{array}$ \\
\hline B & $2.03(3.32)$ & 1.50 & 12.83 & 0 & -1.21 & - & -0.89 \\
\hline $\mathrm{Al}$ & $2.03(3.29)$ & 1.81 & 12.99 & 0 & -2.31 & - & -1.46 \\
\hline In & $1.80(3.09)$ & 2.06 & 13.11 & 0 & 1.26 & - & -1.46 \\
\hline $\mathrm{C}$ & $1.82(2.92)$ & 1.40 & 12.84 & 0 & 1.32 & - & -0.35 \\
\hline $\mathrm{Si}$ & $1.87(3.04)$ & 1.70 & 12.98 & 0 & -0.86 & - & -1.67 \\
\hline $\mathrm{Ge}$ & $1.76(2.90)$ & 1.83 & 13.05 & 0 & 1.11 & - & -1.33 \\
\hline $\mathrm{P}$ & $0.29(0.52)$ & 1.64 & 13.01 & 0 & 1.26 & - & -1.42 \\
\hline As & $0.63(1.23)$ & 1.91 & 13.08 & 0 & 2.52 & - & -1.34 \\
\hline $\mathrm{Sb}$ & $0.69(1.22)$ & 2.05 & 13.15 & 0 & 2.73 & - & -1.71 \\
\hline \multirow[t]{2}{*}{$\mathrm{O}$} & Up $1.36(2.84)$ & 1.20 & 13.08 & 3 & 5.79 & 0.39 & +0.42 \\
\hline & Dn 0.59 (1.92) & & & & & & \\
\hline \multirow[t]{2}{*}{ S } & Up 1.07 (1.91) & 1.87 & 13.04 & 1 & 4.25 & 0.15 & -0.70 \\
\hline & Dn $0.46(1.04)$ & & & & & & \\
\hline \multirow[t]{2}{*}{$\mathrm{Se}$} & Up 0.94 (2.09) & 1.99 & 13.09 & 1 & 3.93 & 0.14 & -0.89 \\
\hline & Dn $0.76(1.41)$ & & & & & & \\
\hline
\end{tabular}



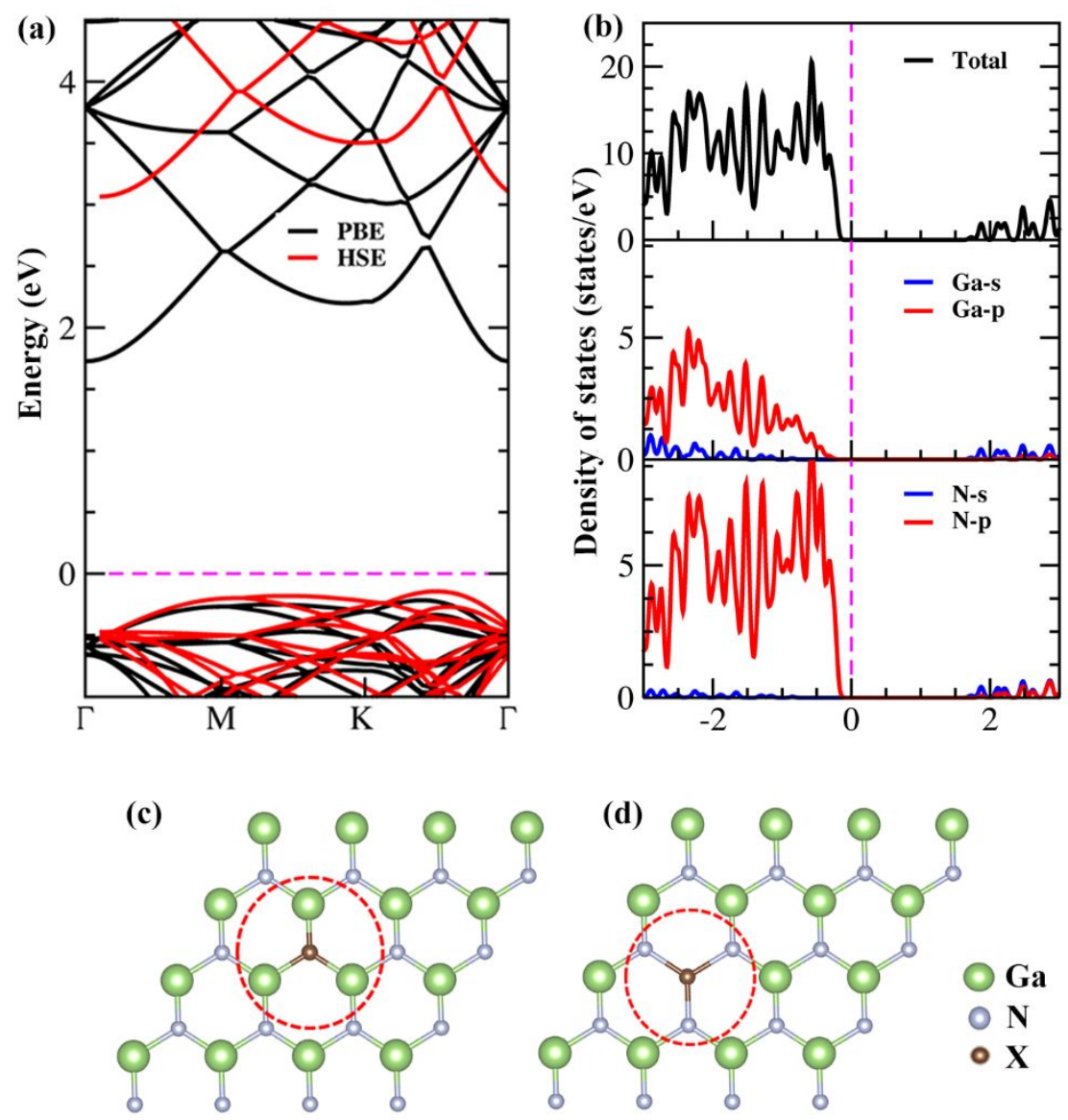

Fig. 1. (a) DFT-PBE and HSE band structures of pure 2D GaN ML (4×4 supercell). (b) PDOS of pure g$\mathrm{GaN}$. Geometric structures of X-doped g-GaN ML at (c) N-site and (d) Ga-site. Dashed red circles show that the dopant has three nearest $\mathrm{Ga} / \mathrm{N}$ atoms under $\mathrm{Ga}-\mathrm{N}$-rich conditions, respectively. 

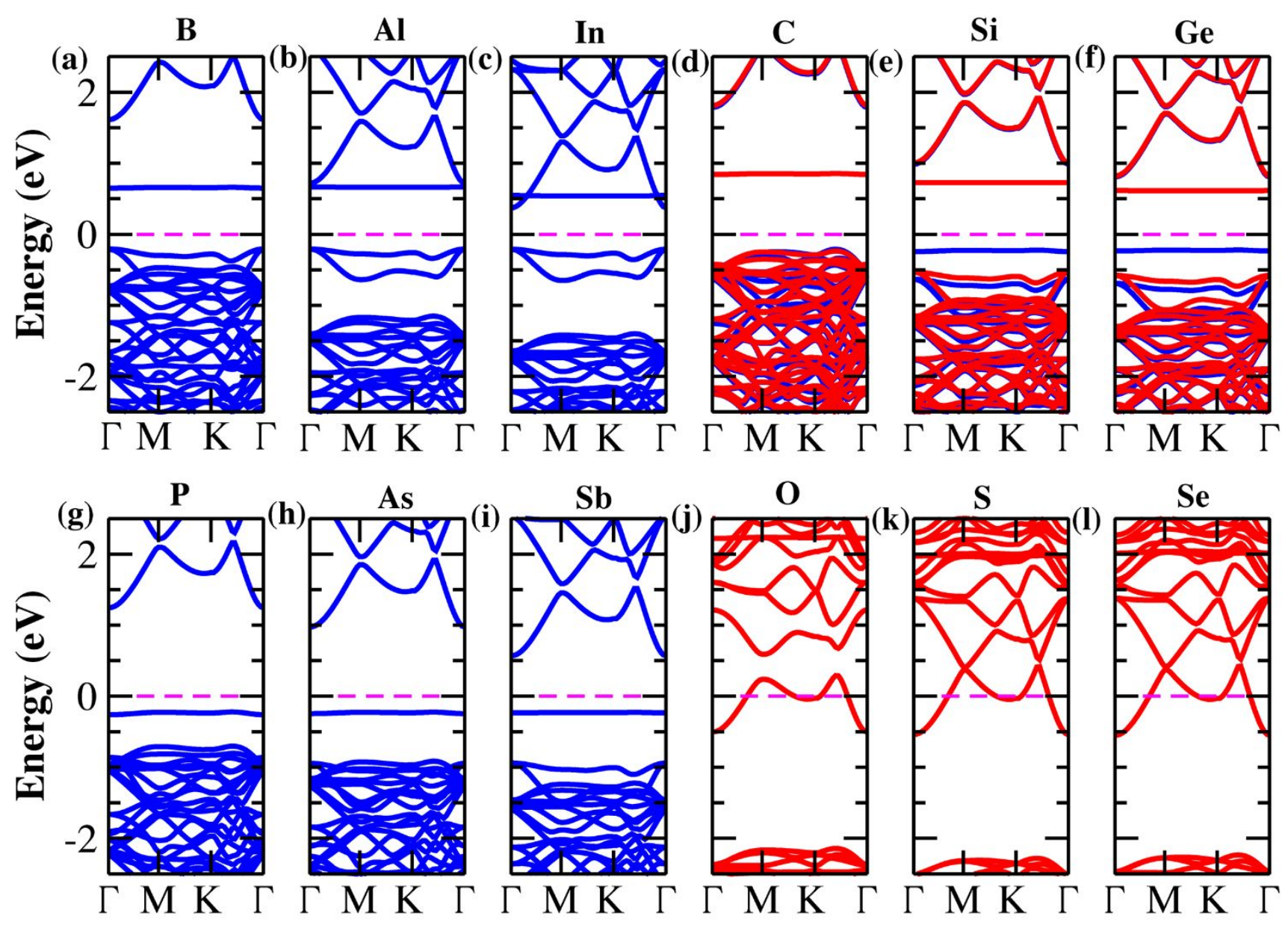

Fig. 2. DFT-PBE band structures of X-doped GaN under Ga-rich conditions. The pink dashed line represents the Fermi level. In (a)-(c) and (g) - (i), blue lines indicate semiconducting behavior. In (d)-(f), red and blue lines indicate band structures that represent band splitting with spin-up (red) and spin-down (blue) channels, respectively. In (j)-(l), red line structures represent n-type behavior. 


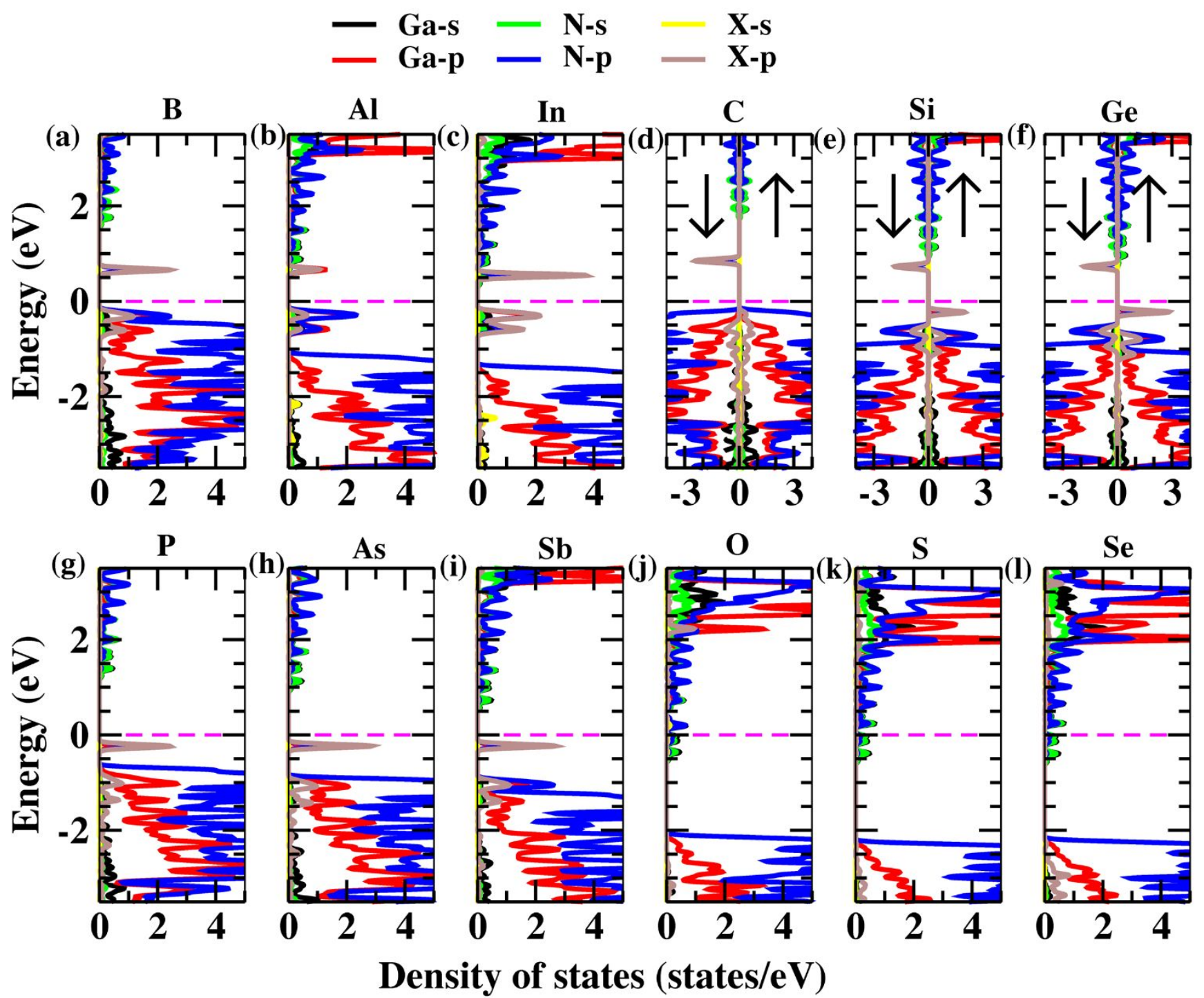

Fig. 3. PDOS of X-doped GaN under Ga-rich conditions. Black and red lines correspond to Ga-s and Ga- $p$ states, green and blue lines correspond to $\mathrm{N}-s$ and $\mathrm{N}-p$ states, and yellow and brown color correspond to $\mathrm{X}-s$ and $\mathrm{X}-p$ states. Up and down arrows denote spin-up and spin-down channels, respectively. 
Ga-rich

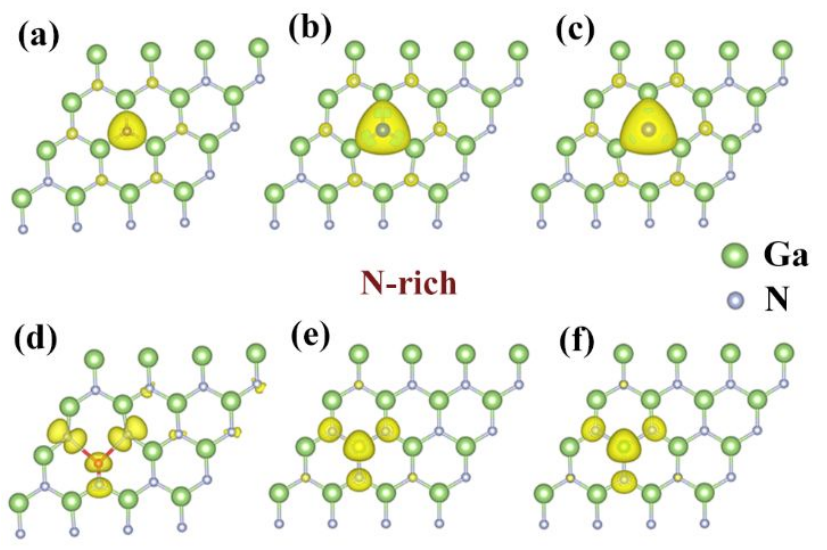

Fig.4. Spin-density plots of (a) C-, (b) Si- and (c) Ge-doped g-GaN under Ga-rich conditions. Spin density plots of (d) O-, (e) S- and (f) Se-doped g-GaN in N-rich conditions. The yellow color represents the contribution to the magnetic moment.

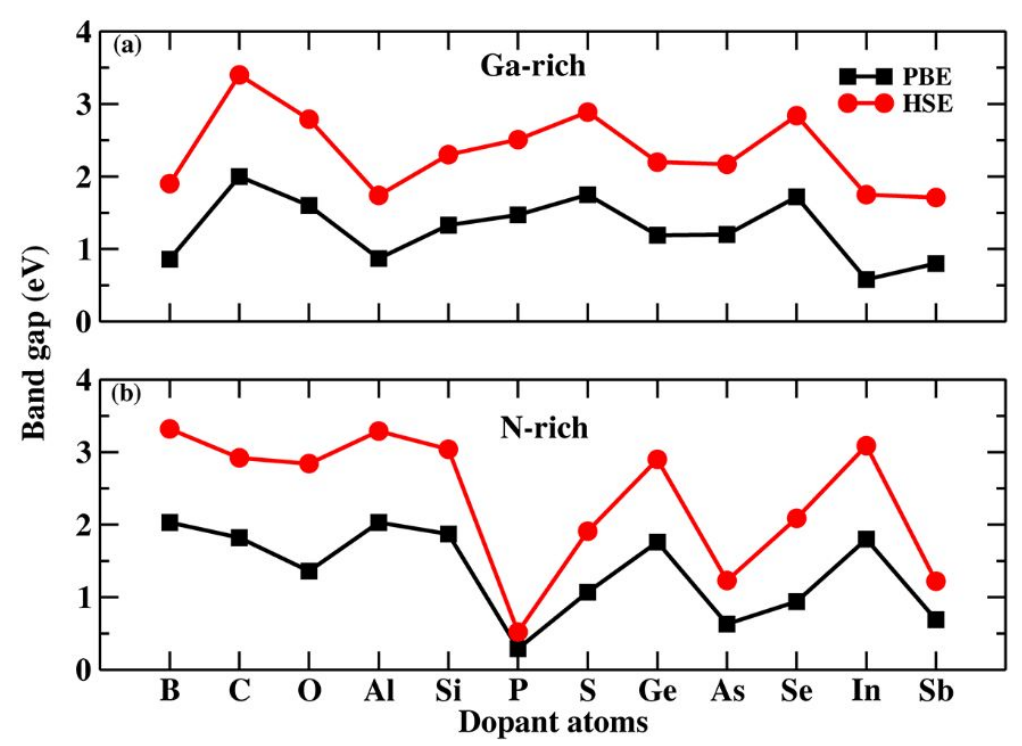

Fig. 5. PBE and HSE band gaps of doped g-GaN MLs as a function of various dopants under (a) Ga-rich and (b) N-rich conditions at 3.125\% dopant concentration. It should be noted that for spin-polarized configurations, only larger band gap values are considered here. 

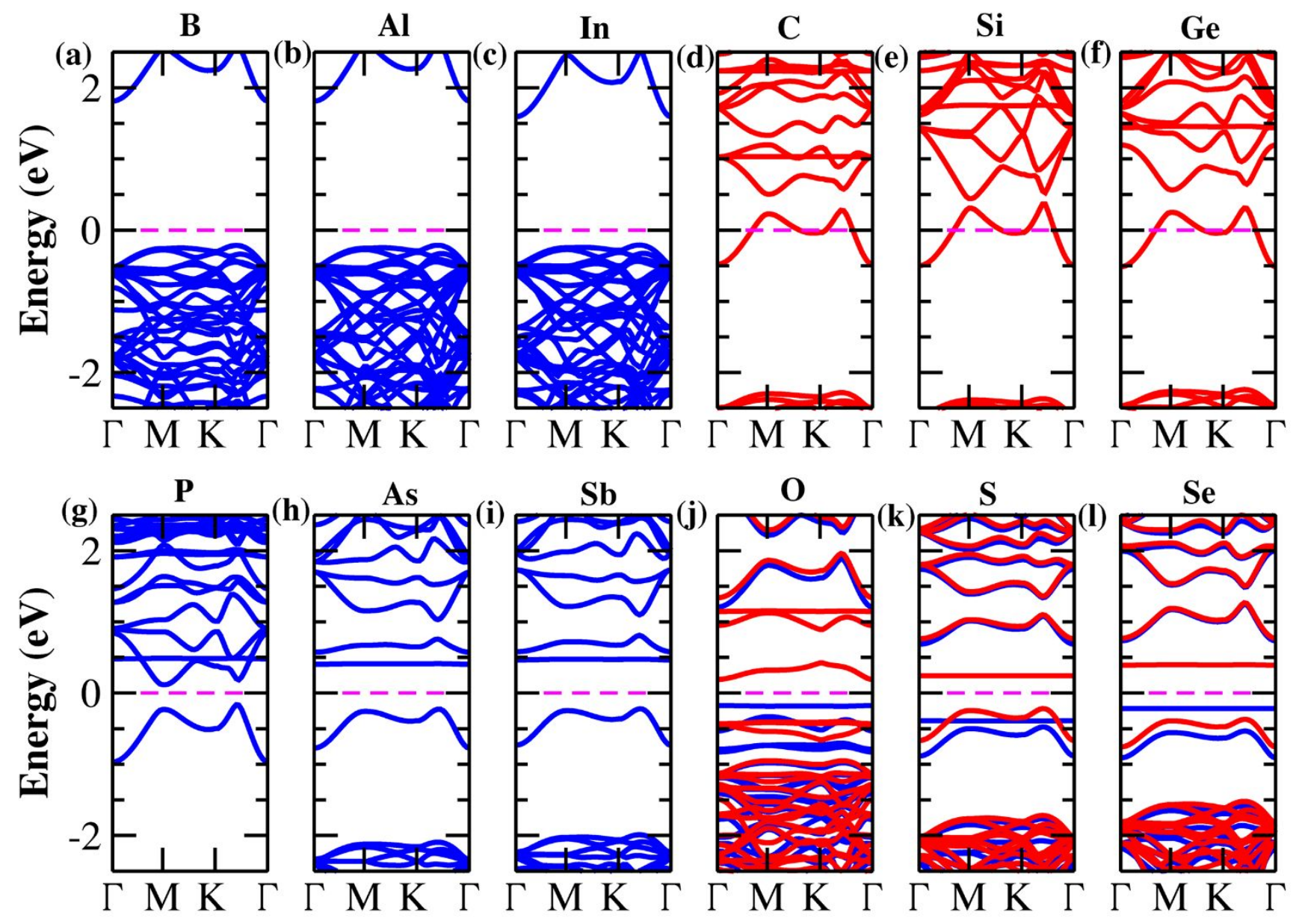

Fig. 6. DFT-PBE band structures of X-doped GaN under N-rich conditions. In (a)-(c) and (g)-(i) blue lines indicate semiconductor behavior. In (j)-(1) red and blue lines represent band structures denoting spin-up and spin-down channels. In (d)-(f) red lines represent n-type behavior. 

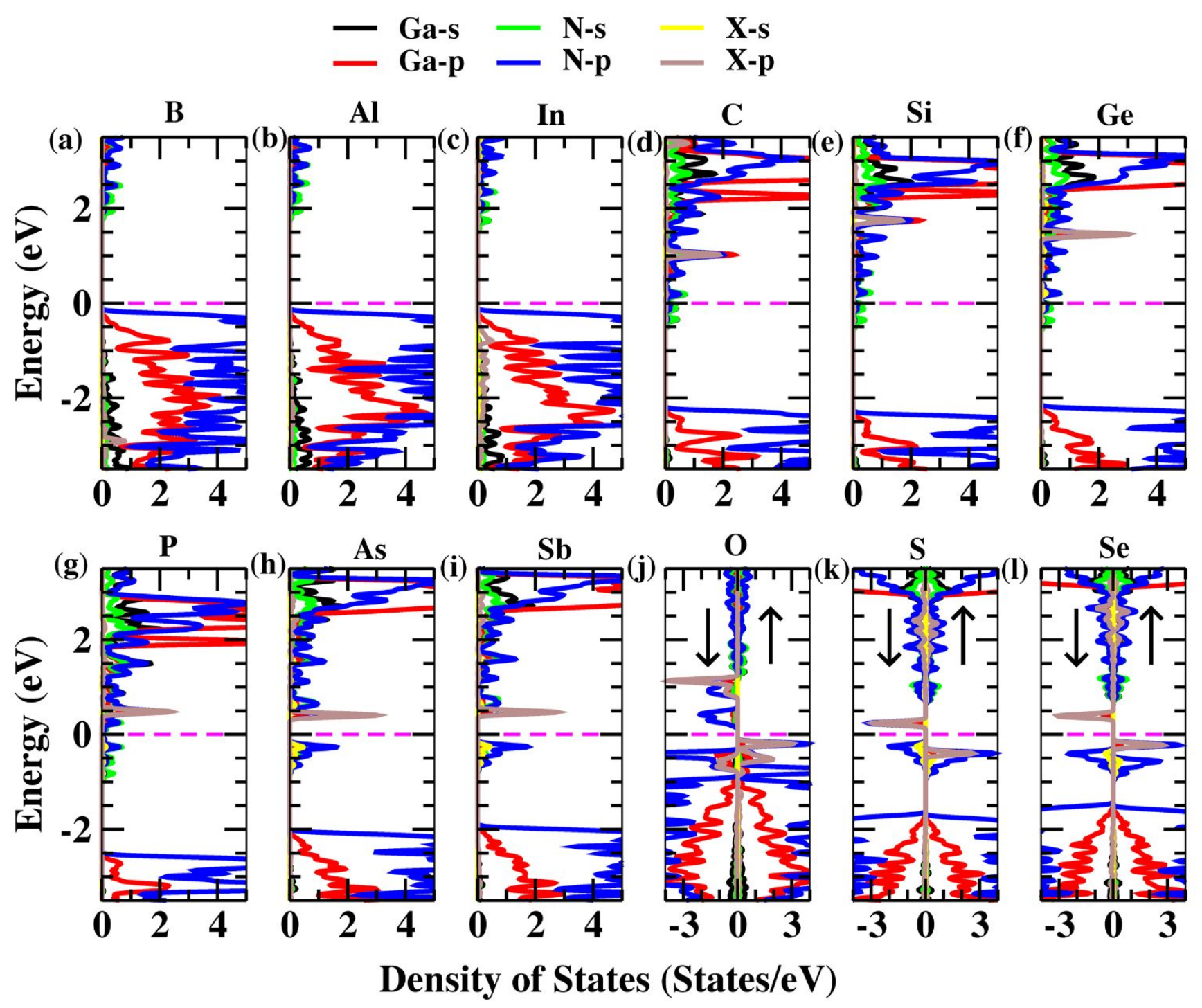

Fig. 7. PDOS of X-doped GaN under the N-rich conditions. Black and red lines correspond to Ga-s and Ga- $p$, green and blue lines correspond to $\mathrm{N}-s$ and $\mathrm{N}-p$, and yellow and brown lines correspond to $\mathrm{X}-s$ and $\mathrm{X}-p$. 

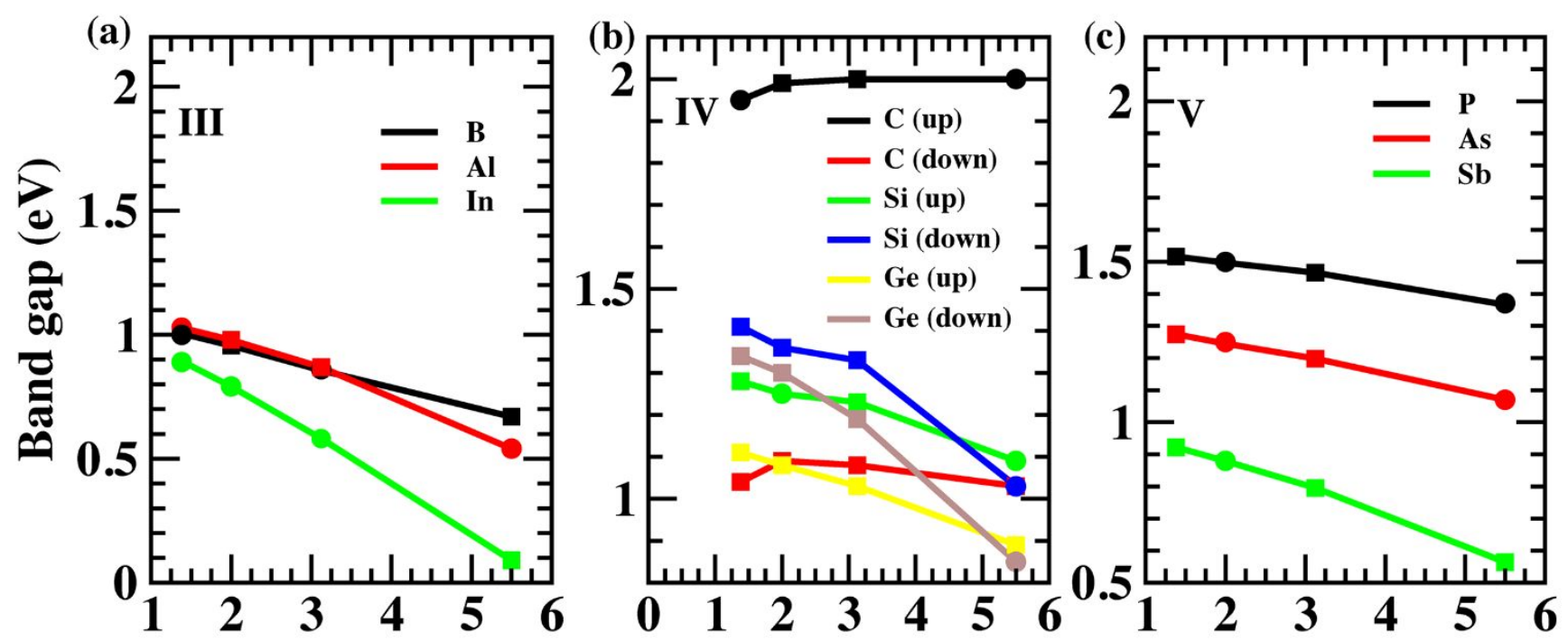

Dopant concentration $(\%)$

Fig. 8. The band gap behavior of the $\mathrm{X}$-doped $\mathrm{g}-\mathrm{GaN}$ as a function of $\mathrm{X}$ concentration under Ga-rich conditions. The filled circles and squares indicate direct and indirect band gaps, respectively.

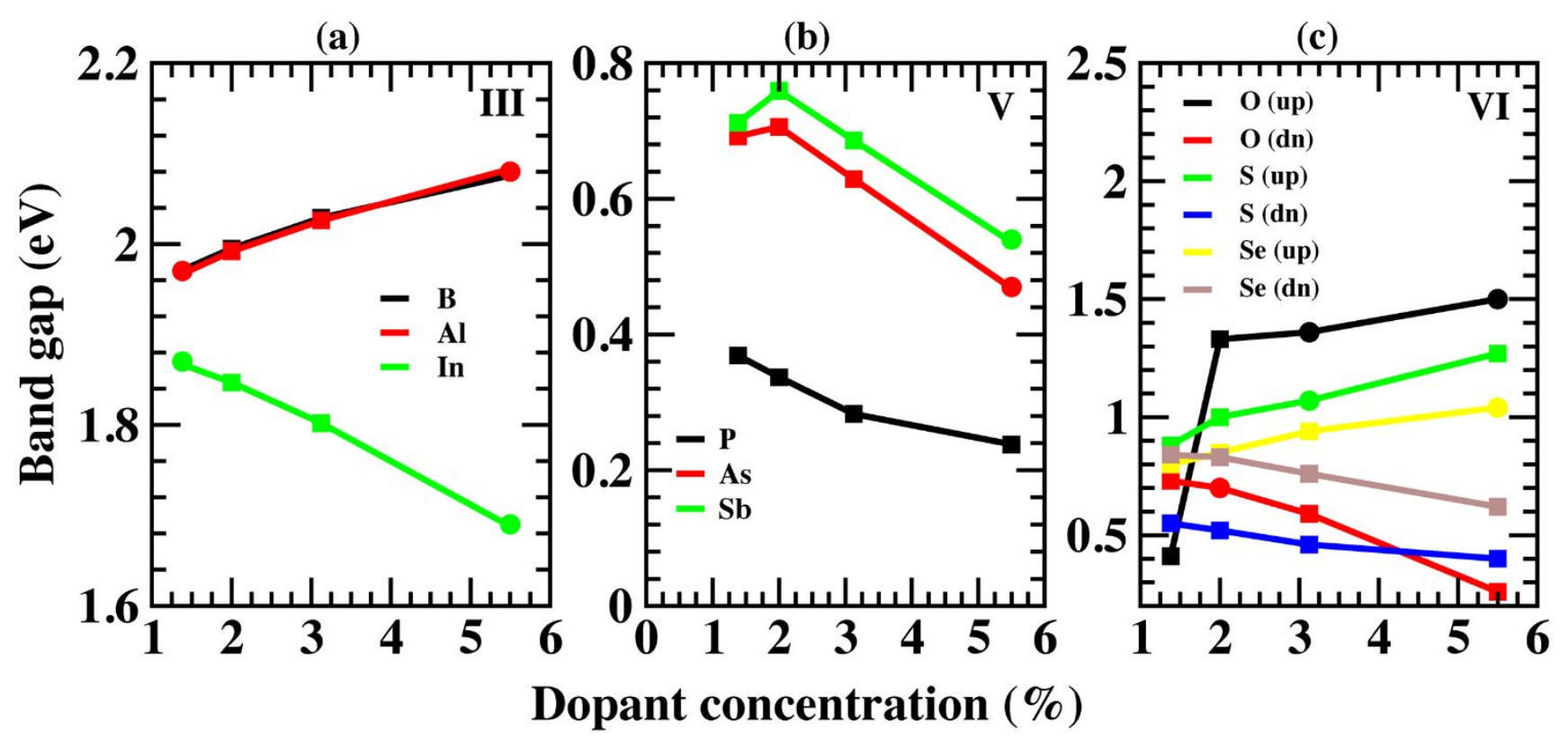

Fig. 9. The band gap behavior of the $\mathrm{X}$-doped $\mathrm{g}-\mathrm{GaN}$ as a function of $\mathrm{X}$ concentration under $\mathrm{N}$-rich conditions. The filled circles and squares indicate direct and indirect band gaps, respectively. 

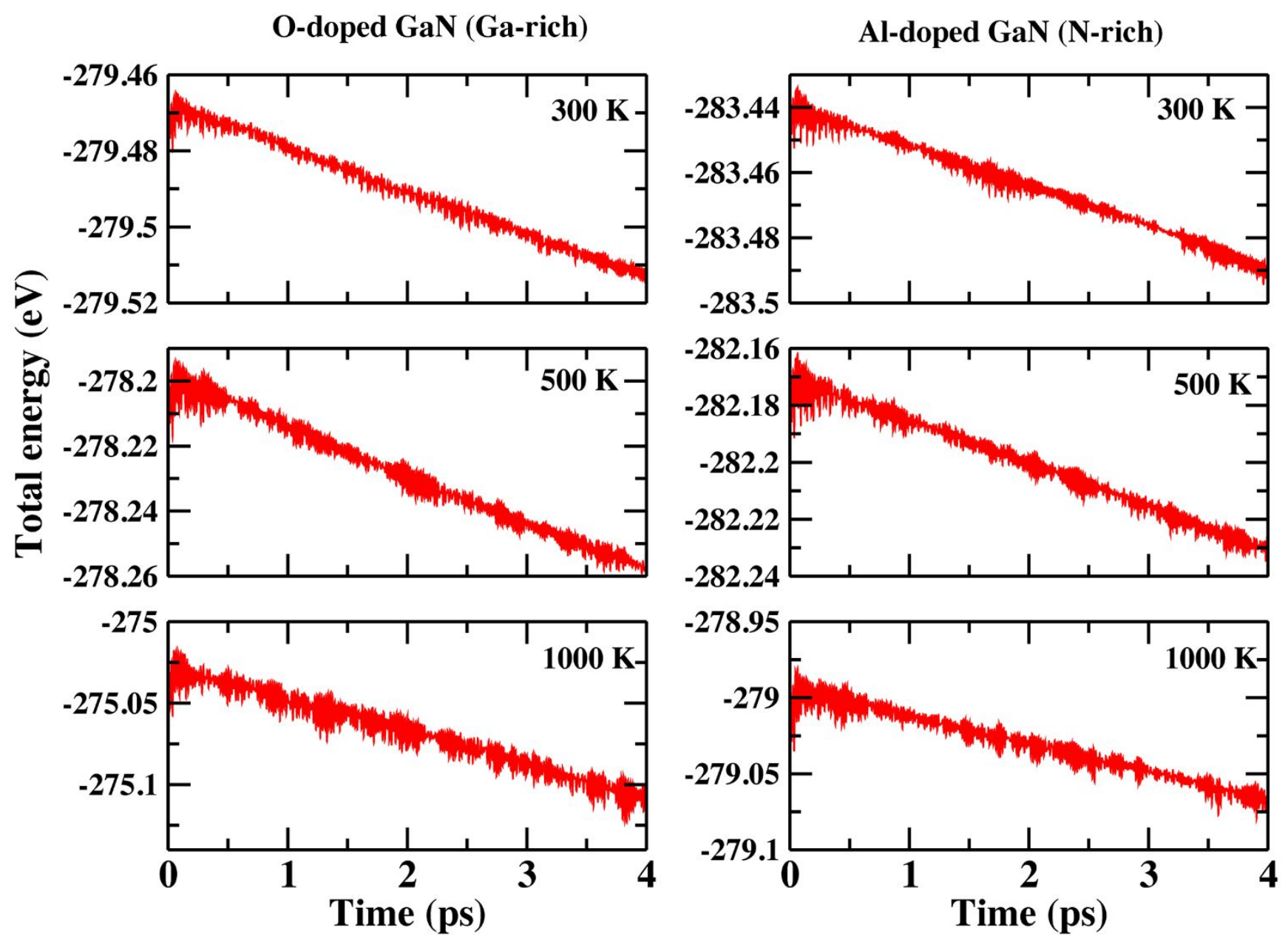

Fig. 10. The total energy fluctuations of O-doped GaN and A-doped GaN as a function of simulated time in Ga- and N-rich conditions, respectively, at three temperatures $(300 \mathrm{~K}, 500 \mathrm{~K}$ and $1000 \mathrm{~K})$. 
TOC

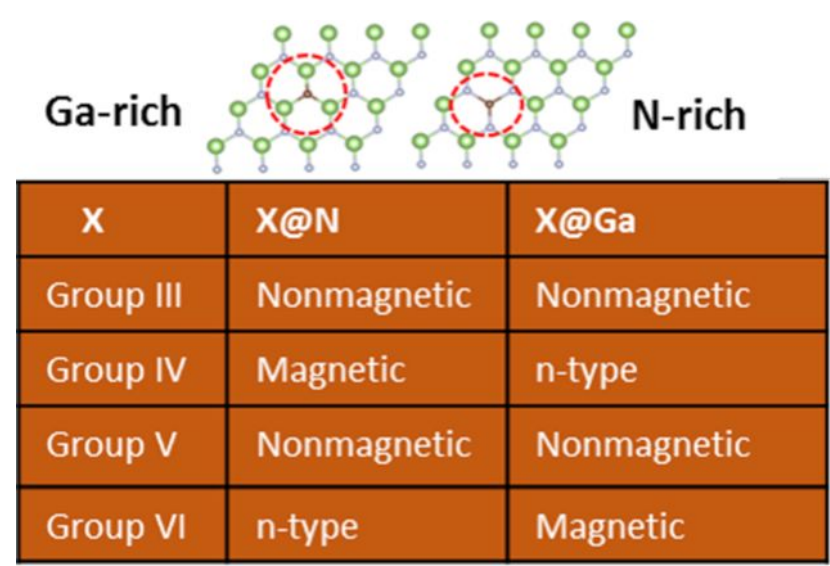



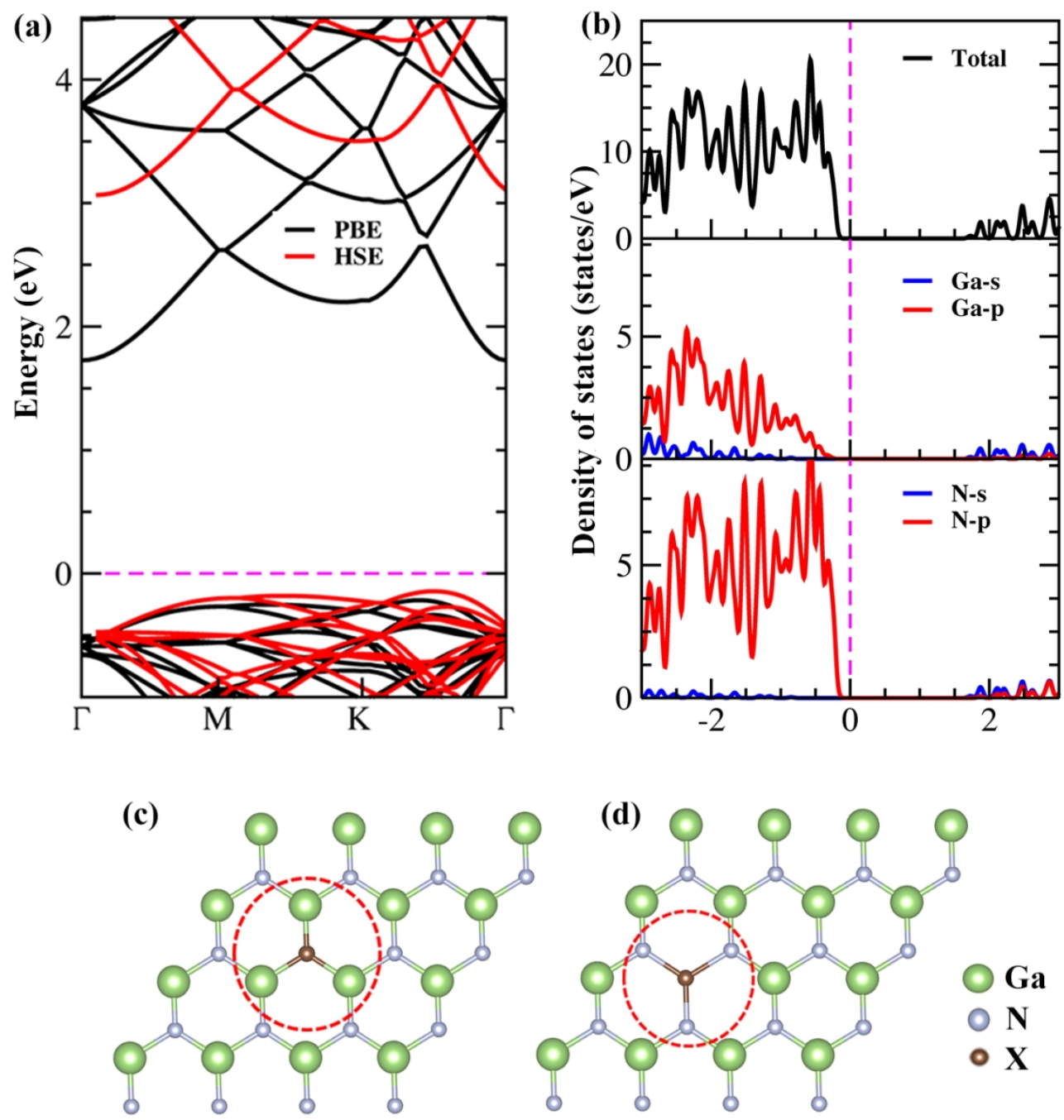

Fig. 1. (a) DFT-PBE and HSE band structures of pure 2D GaN ML ( $4 \times 4$ supercell). (b) PDOS of pure g-GaN. Geometric structures of X-doped g-GaN ML at (c) N-site and (d) Ga-site. Dashed red circles show that the dopant has three nearest $\mathrm{Ga} / \mathrm{N}$ atoms under $\mathrm{Ga}-/ \mathrm{N}-$ rich conditions, respectively. 

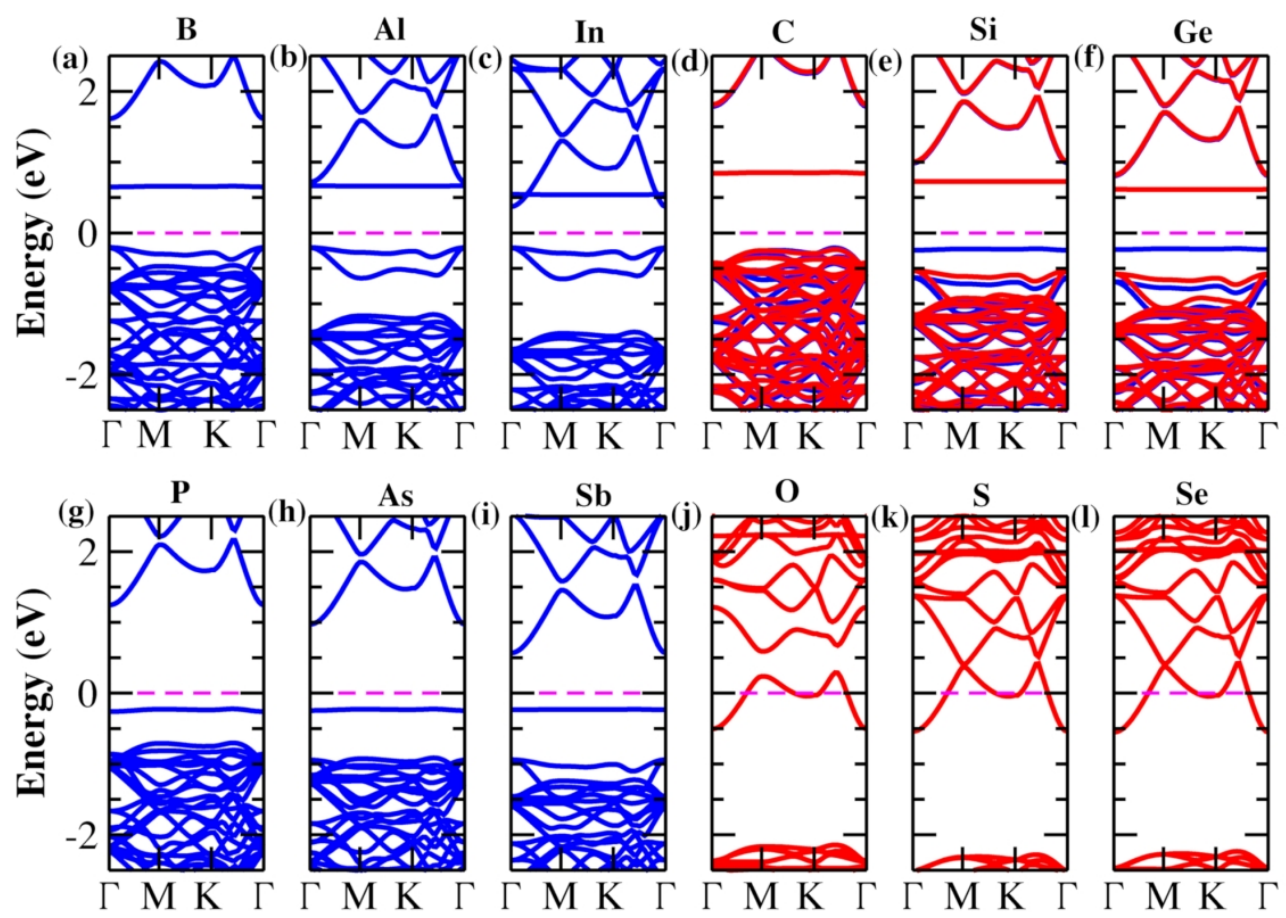

Fig. 2. DFT-PBE band structures of X-doped GaN under Ga-rich conditions. The pink dashed line rep-resents the Fermi level. In (a)-(c) and (g) - (i), blue lines indicate semiconducting behavior. In (d) - ( f), red and blue lines indicate band structures that represent band splitting with spin-up (red) and spin-down (blue) channels, respectively. In $(j)-(I)$, red line structures represent $n$-type behavior.

$170 \times 123 \mathrm{~mm}(300 \times 300 \mathrm{DPI})$ 


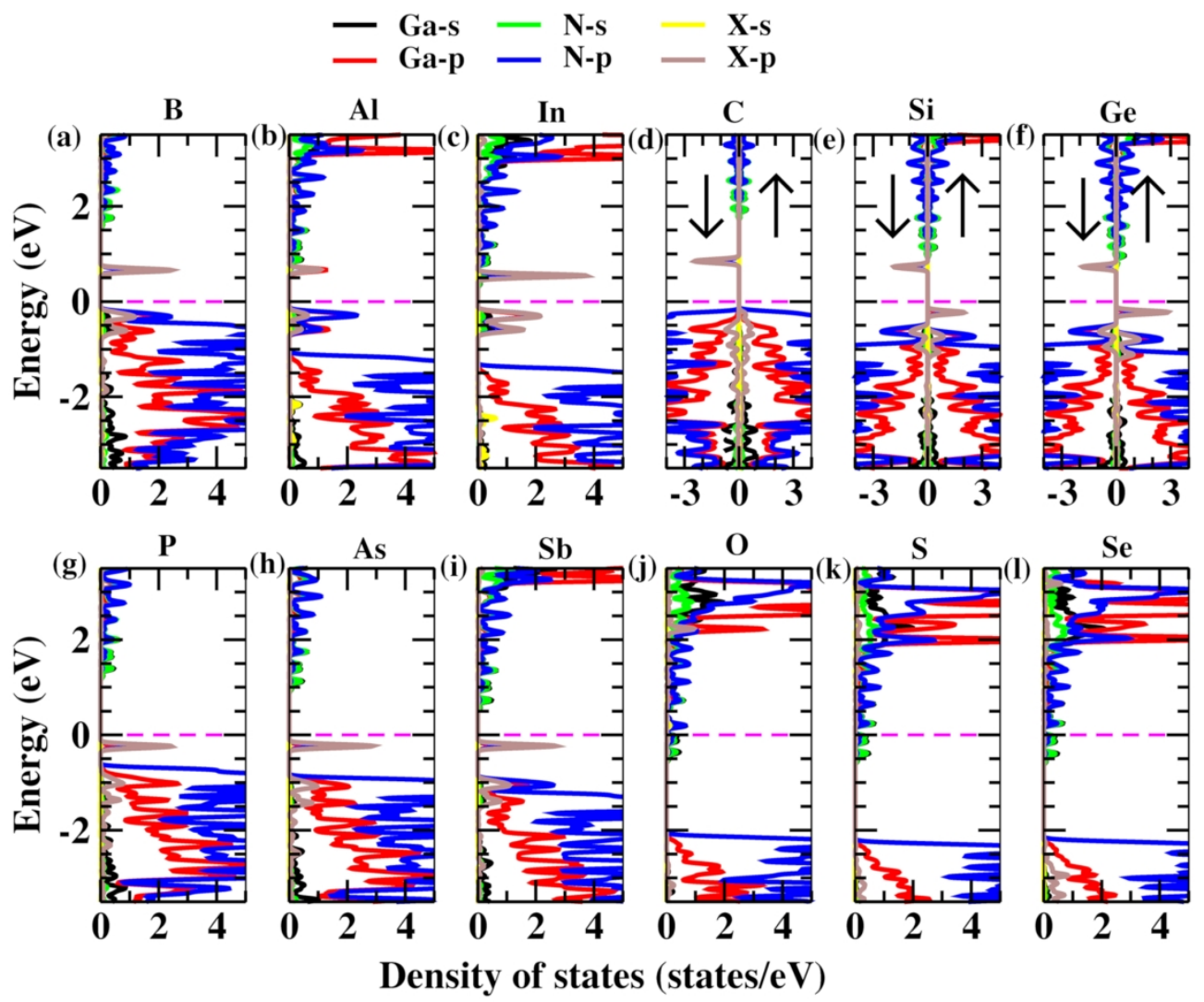

Fig. 3. PDOS of X-doped GaN under Ga-rich conditions. Black and red lines correspond to Ga-s and Ga-p states, green and blue lines correspond to N-s and N-p states, and yellow and brown color correspond to X-s and X-p states. Up and down arrows denote spin-up and spin-down channels, respectively.

$170 \times 141 \mathrm{~mm}(300 \times 300 \mathrm{DPI})$ 


\section{Ga-rich}

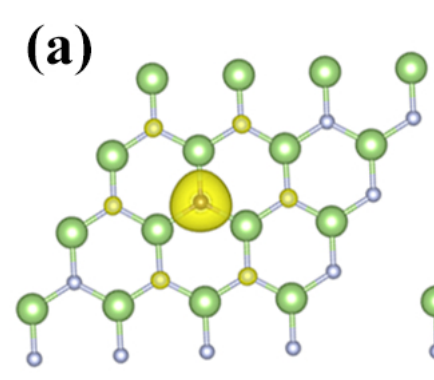

(b)

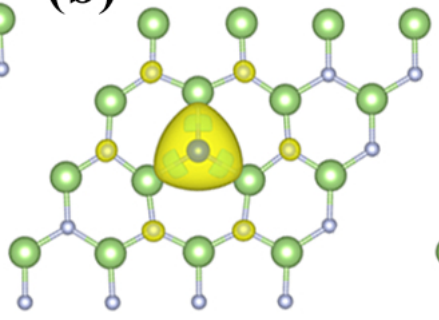

N-rich

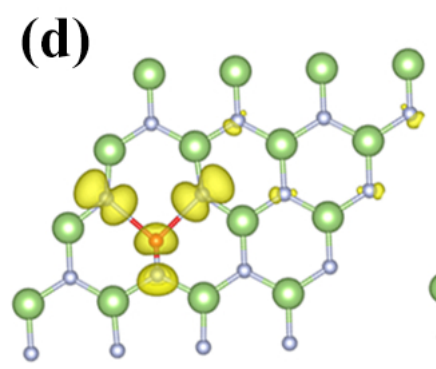

(e)

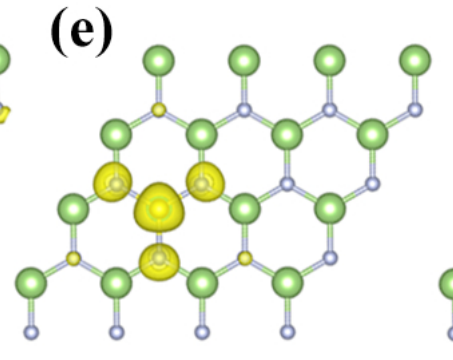

(c)

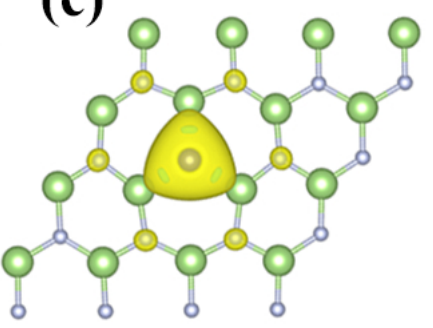

- Ga ○ $\mathbf{N}$

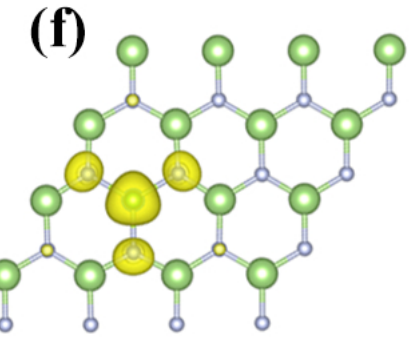

Fig.4. Spin-density plots of (a) C-, (b) Si- and (c) Ge-doped g-GaN under Ga-rich conditions. Spin density plots of (d) O-, (e) S- and (f) Se-doped g-GaN in N-rich conditions. The yellow color represents the contribution to the magnetic moment.

$82 \times 61 \mathrm{~mm}(300 \times 300 \mathrm{DPI})$ 


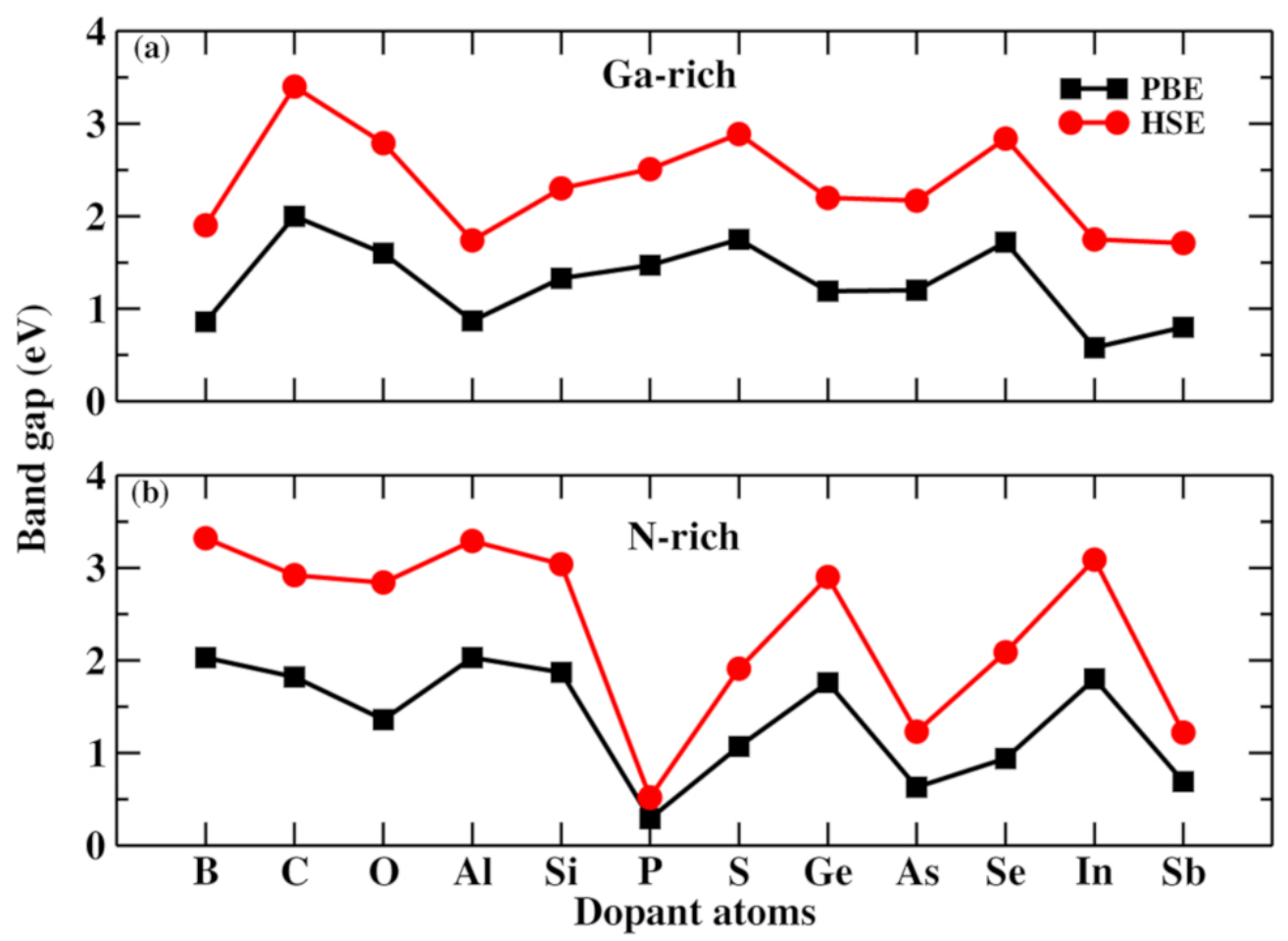

Fig. 5. PBE and HSE band gaps of doped g-GaN MLs as a function of various dopants under (a) Ga-rich and (b) N-rich conditions at 3.125\% dopant concentration. It should be noted that for spin-polarized configurations, only larger band gap values are considered here.

$85 \times 61 \mathrm{~mm}(300 \times 300 \mathrm{DPI})$ 

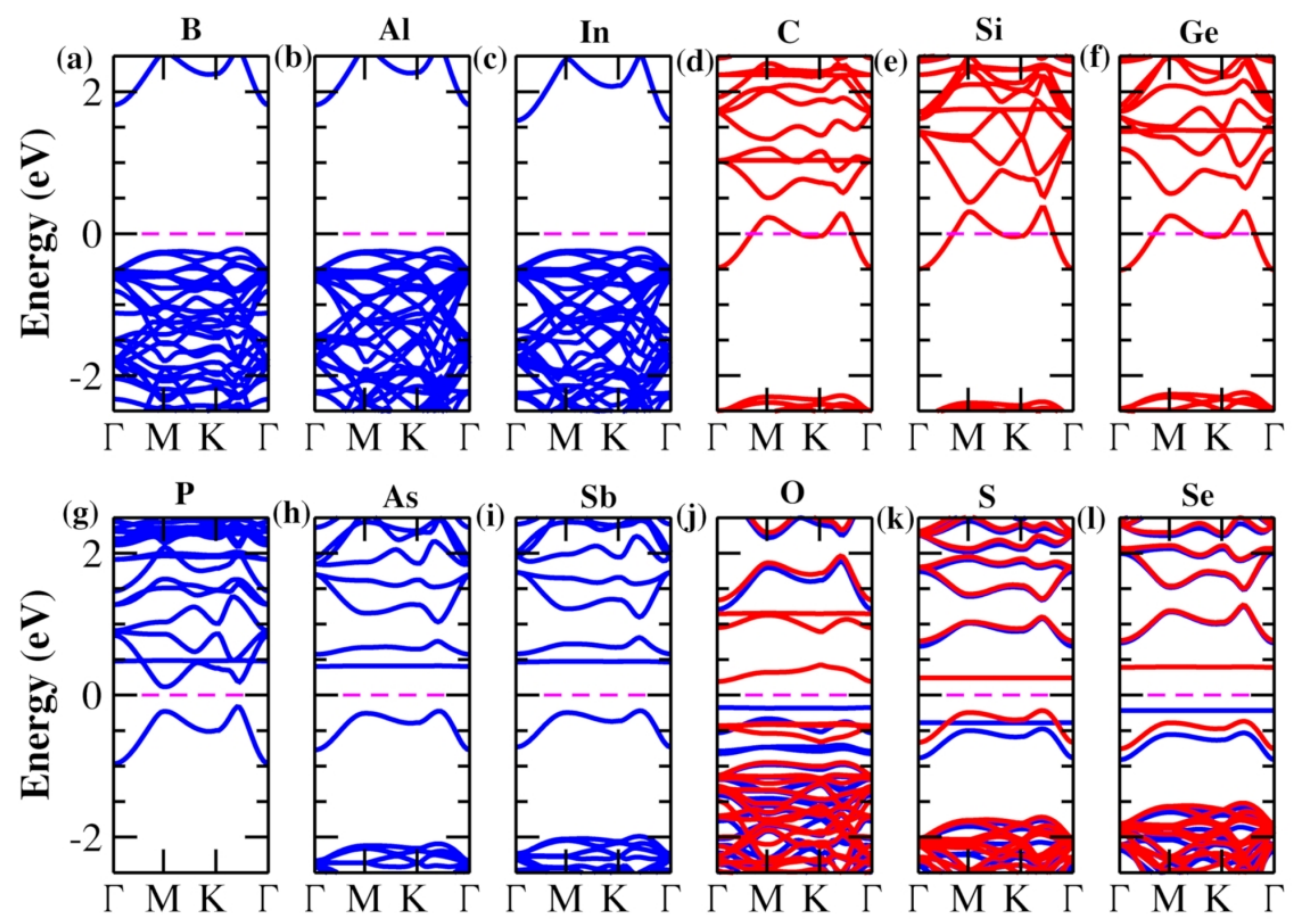

Fig. 6. DFT-PBE band structures of X-doped GaN under N-rich conditions. In (a)-(c) and (g)-(i) blue lines indicate semiconductor behavior. In $(j)-(I)$ red and blue lines represent band structures denoting spin-up and spin-down channels. In (d)-(f) red lines represent n-type behavior.

$170 \times 121 \mathrm{~mm}(300 \times 300 \mathrm{DPI})$ 

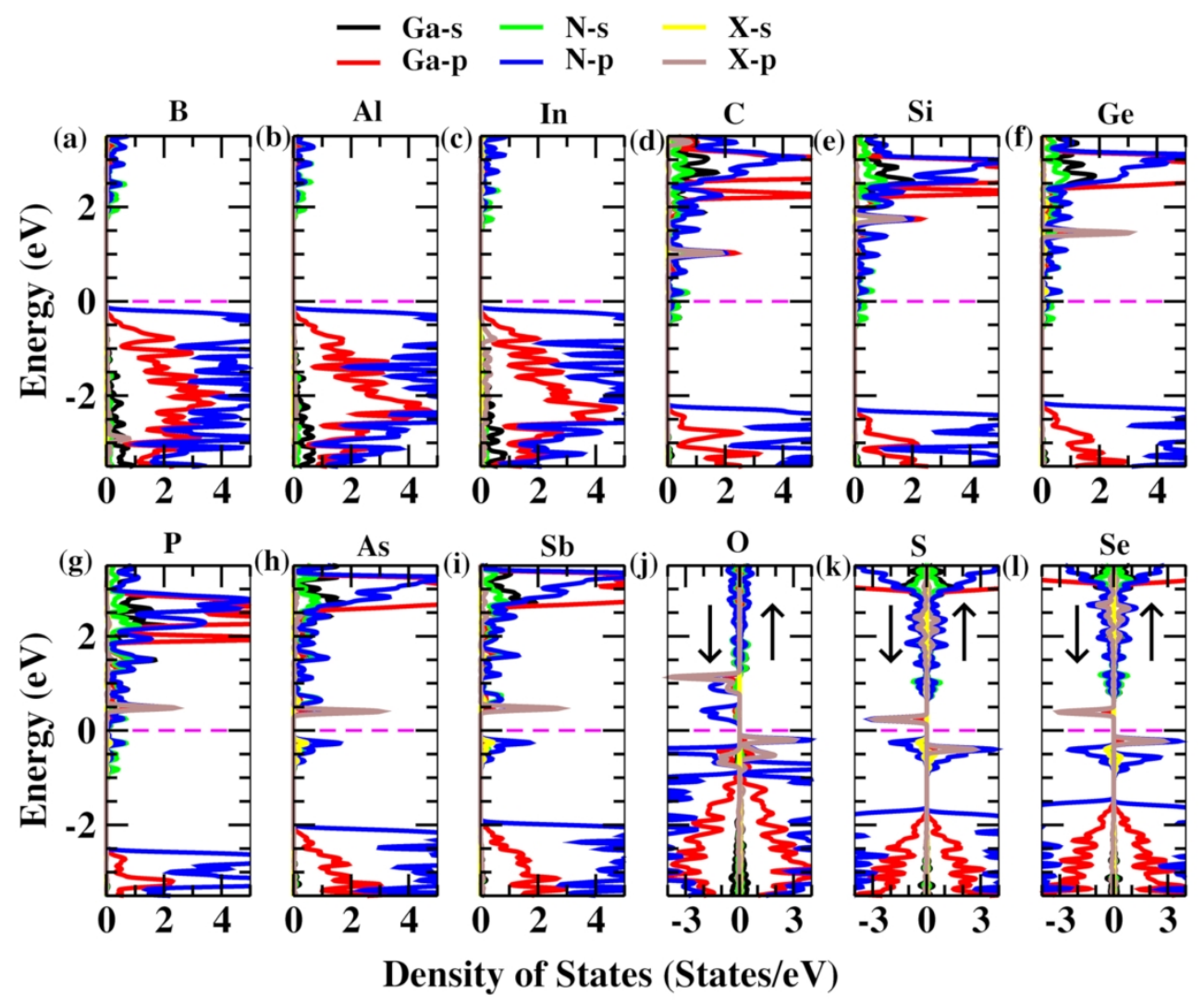

Fig. 7. PDOS of X-doped GaN under the N-rich conditions. Black and red lines correspond to Ga-s and Ga-p, green and blue lines correspond to $\mathrm{N}-\mathrm{s}$ and $\mathrm{N}-\mathrm{p}$, and yellow and brown lines correspond to X-s and X-p.

$170 \times 143 \mathrm{~mm}(300 \times 300 \mathrm{DPI})$ 

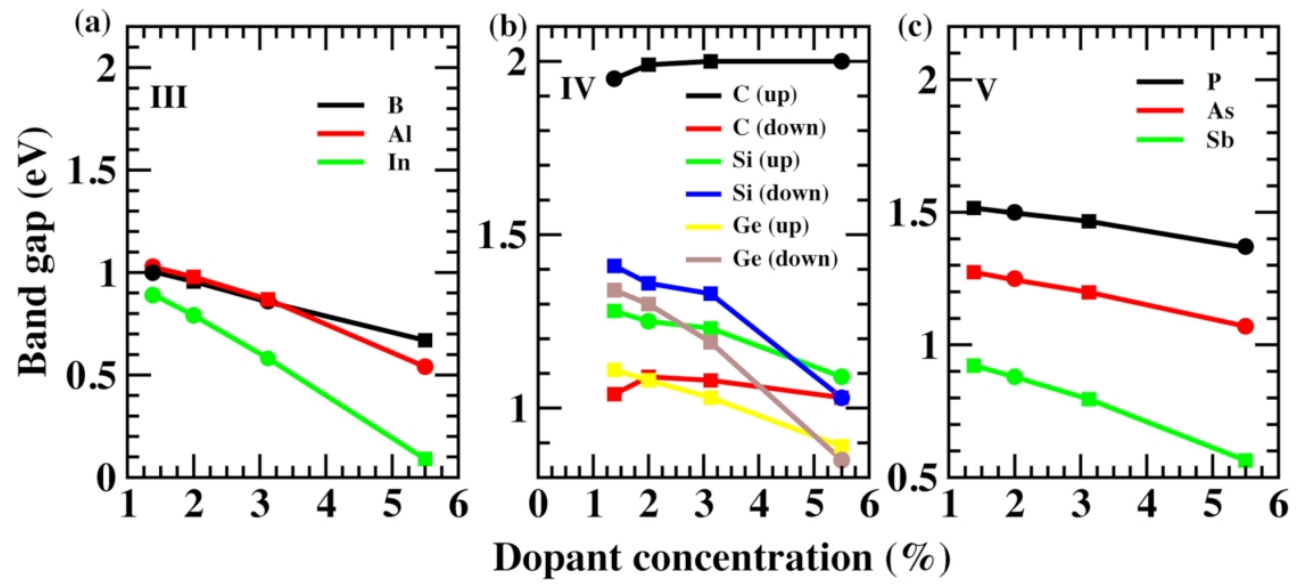

Fig. 8. The band gap behavior of the X-doped $g-G a N$ as a function of $X$ concentration under Ga-rich conditions. The filled circles and squares indicate direct and indirect band gaps, respectively.

$$
170 \times 79 \mathrm{~mm}(300 \times 300 \mathrm{DPI})
$$


2

3

4

5

6

7

8

9

10

11

12

13

14

15

16

17

18

19

20

21

22

23

24

25

26

27

28

29

30

31

32

33

34

35

36

37

38

39

40

41

42

43

44

45

46

47

48

49

50

51

52

53

54

55

56

57

58

59

60
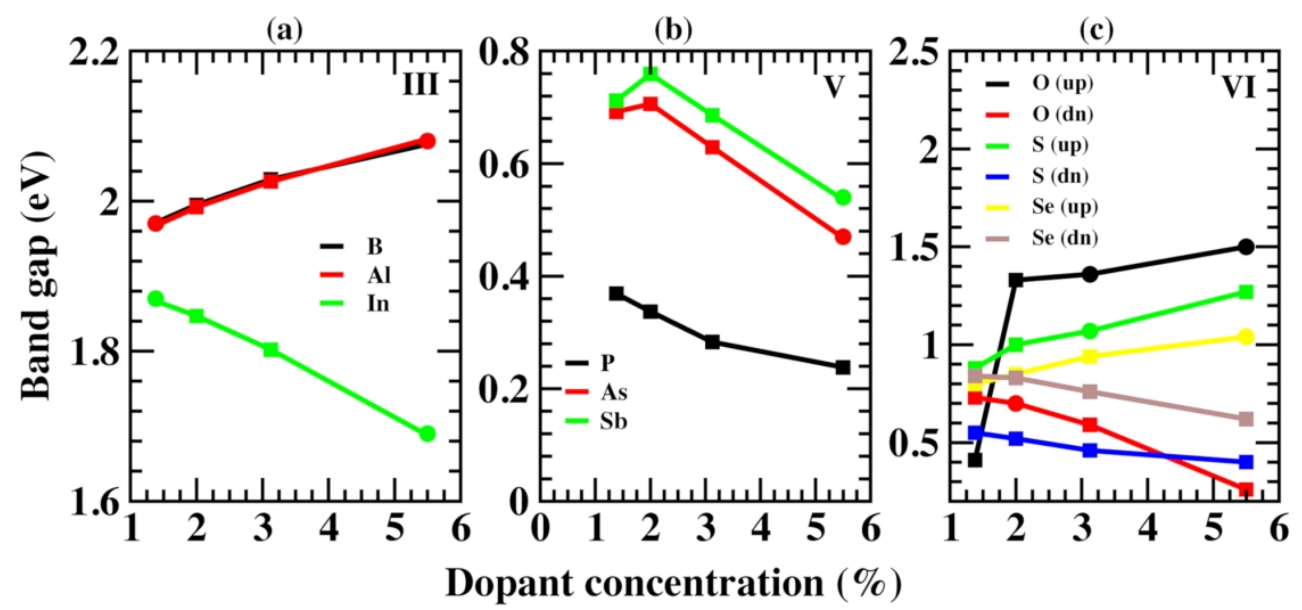

Fig. 9. The band gap behavior of the $X$-doped $g-G a N$ as a function of $X$ concentration under $\mathrm{N}$-rich conditions. The filled circles and squares indicate direct and indirect band gaps, respectively.

$$
170 \times 82 \mathrm{~mm}(300 \times 300 \mathrm{DPI})
$$



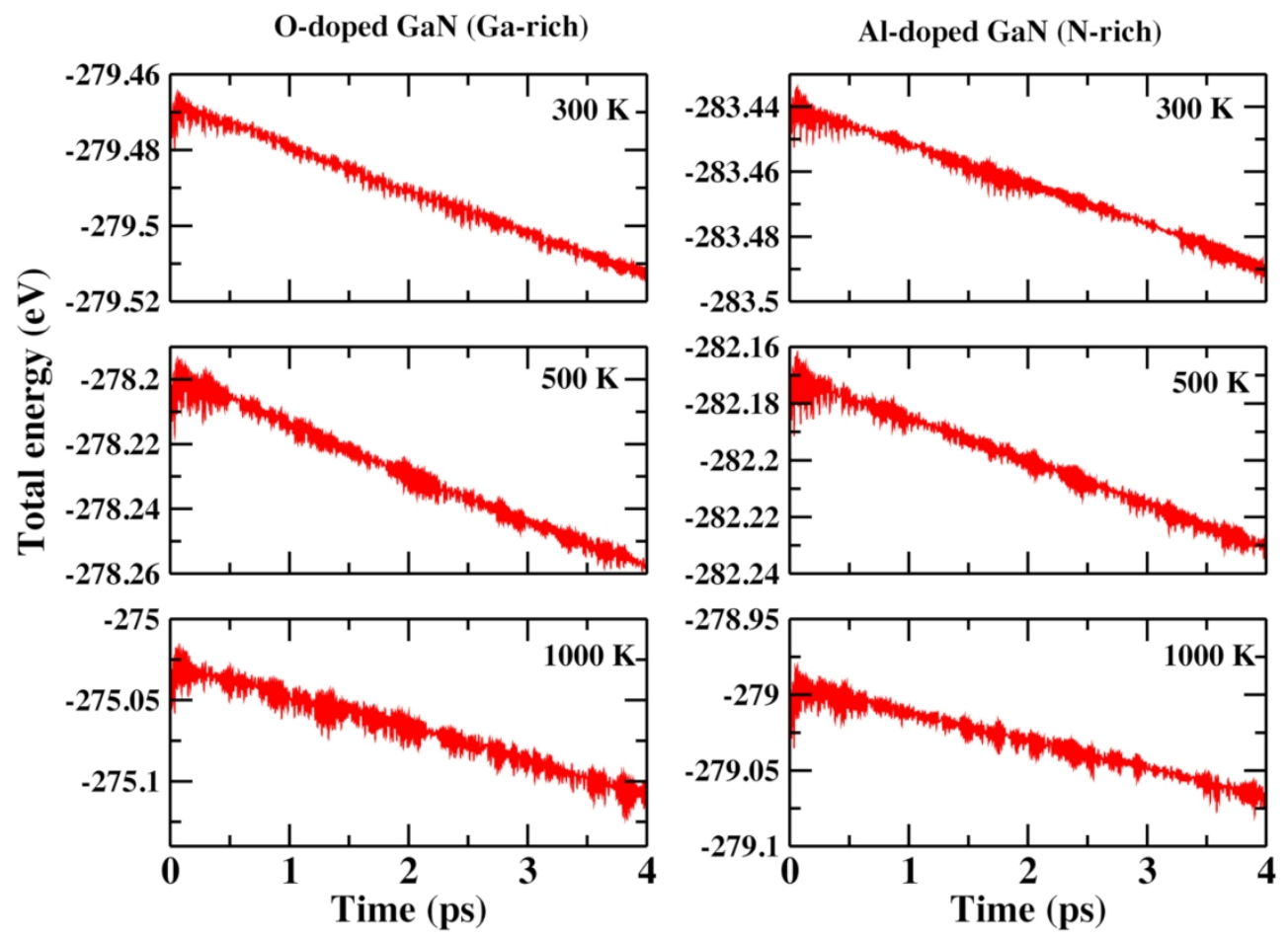

Fig. 10. The total energy fluctuations of O-doped GaN and A-doped GaN as a function of simulated time in Ga- and N-rich conditions, respectively, at three temperatures (300 K, $500 \mathrm{~K}$ and $1000 \mathrm{~K})$.

$170 \times 124 \mathrm{~mm}(300 \times 300 \mathrm{DPI})$ 


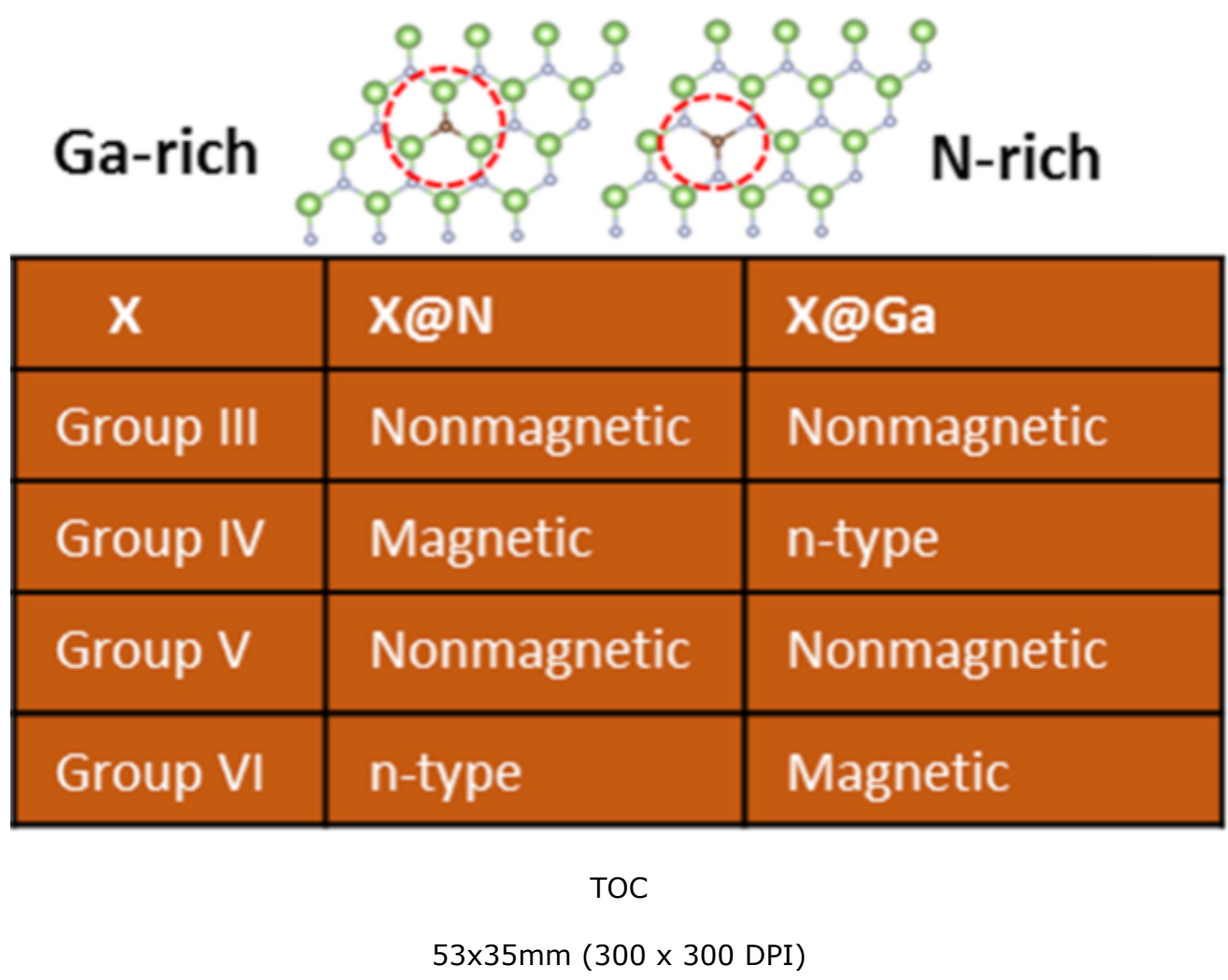

